\title{
Adiponectin/T-cadherin system enhances exosome biogenesis and decreases cellular ceramides by exosomal release
}

\author{
Yoshinari Obata, ${ }^{1}$ Shunbun Kita,,${ }^{1,2}$ Yoshihisa Koyama, ${ }^{3}$ Shiro Fukuda, ${ }^{1}$ Hiroaki Takeda, ${ }^{4}$ \\ Masatomo Takahashi, ${ }^{4}$ Yuya Fujishima, ${ }^{1}$ Hirofumi Nagao, ${ }^{1}$ Shigeki Masuda, ${ }^{1}$ Yoshimitsu Tanaka, \\ Yuto Nakamura, ${ }^{1}$ Hitoshi Nishizawa, ${ }^{1}$ Tohru Funahashi, ${ }^{1,5}$ Barbara Ranscht, ${ }^{6}$ Yoshihiro Izumi, ${ }^{4}$ \\ Takeshi Bamba, ${ }^{4}$ Eiichiro Fukusaki, ${ }^{7}$ Rikinari Hanayama, ${ }^{8}$ Shoichi Shimada, ${ }^{3}$ Norikazu Maeda, ${ }^{1,5}$ \\ and lichiro Shimomura ${ }^{1}$ \\ 'Department of Metabolic Medicine, 'Department of Adipose Management, and ${ }^{3}$ Neuroscience and Cell Biology, Graduate \\ School of Medicine, Osaka University, Osaka, Japan. ${ }^{4}$ Division of Metabolomics, Medical Institute of Bioregulation, \\ Kyushu University, Fukuoka, Japan. ${ }^{5}$ Department of Metabolism and Atherosclerosis, Graduate School of Medicine, \\ Osaka University, Osaka, Japan. ${ }^{6}$ Sanford Burnham Prebys Medical Discovery Institute, NIH-designated Cancer Center, \\ Development, Aging and Regeneration Program, La Jolla, California, USA. 'Department of Biotechnology, Graduate School \\ of Engineering, Osaka University, Osaka, Japan. ${ }^{8}$ Department of Immunology, Kanazawa University Graduate School of \\ Medical Sciences, Ishikawa, Japan.
}

\begin{abstract}
Adiponectin, an adipocyte-derived circulating protein, accumulates in vasculature, heart, and skeletal muscles through interaction with a unique glycosylphosphatidylinositol-anchored cadherin, T-cadherin. Recent studies have demonstrated that such accumulation is essential for adiponectin-mediated cardiovascular protection. Here, we demonstrate that the adiponectin/Tcadherin system enhances exosome biogenesis and secretion, leading to the decrease of cellular ceramides. Adiponectin accumulated inside multivesicular bodies, the site of exosome generation, in cultured cells and in vivo aorta, and also in exosomes in conditioned media and in blood, together with T-cadherin. The systemic level of exosomes in blood was significantly affected by adiponectin or T-cadherin in vivo. Adiponectin increased exosome biogenesis from the cells, dependently on T-cadherin, but not on AdipoR1 or AdipoR2. Such enhancement of exosome release accompanied the reduction of cellular ceramides through ceramide efflux in exosomes. Consistently, the ceramide reduction by adiponectin was found in aortas of WT mice treated with angiotensin II, but not in T-cadherin-knockout mice. Our findings provide insights into adiponectin/T-cadherin-mediated organ protection through exosome biogenesis and secretion.
\end{abstract}

Conflict of interest: $S K, Y O, N M$, and IS are named as inventors on a patent claim filed by Osaka University regarding adiponectin-mediated stimulation of exosome production (Japanese Patent Application 2016-015081).

Submitted: January 5, 2018 Accepted: March 14, 2018 Published: April 19, 2018

\section{Reference information:} JCI Insight. 2018;3(8):e99680. https://doi.org/10.1172/jici. insight.99680.

\section{Introduction}

Adiponectin is a circulating protein exclusively produced from adipocytes. It is assembled intracellularly and secreted as trimers, hexamers, and high-molecular-weight multimers $(1,2)$. Clinical analyses have increasingly demonstrated that high-molecular-weight multimeric adiponectin is the active form and exerts various pleiotropic effects (3-6). Recent studies have shown that the active forms, multimeric and hexameric adiponectin, accumulate in tissues such as heart, vascular endothelium, and skeletal muscles through interaction with T-cadherin, a unique glycosylphosphatidylinositol-anchored (GPI-anchored) cadherin (7-9). Importantly, we recently showed that adiponectin suppressed neointimal and atherosclerotic plaque formations through T-cadherin (10). Other groups have also shown the significance of adiponectin/T-cadherin association in cardiovascular protection $(7,8)$. Furthermore, single-nucleotide polymorphism near the T-cadherin gene correlates strongly with plasma adiponectin level and cardiovascular diseases in human subjects (11-16). These emerging pieces of evidence suggest that T-cadherin is essential for adiponectin-mediated organ protection. However, T-cadherin is GPI-anchored cadherin without the intracellular or transmembrane domain required for intracellular signaling; hence, the molecular mechanism has not been elucidated.

Exosomes are small vesicles $(30-100 \mathrm{~nm})$ of endosomal origin delimited by a lipid bilayer, and are thought to mediate cell-to-cell communication under normal and pathological conditions by transferring 
active proteins, mRNA, and small noncoding RNAs $(17,18)$. Moreover, exosomal release serves as an alternative disposal pathway to lysosomes (19). Misfolded proteins or several aggregated toxic proteins, such as amyloid $\beta(20)$, tau (21), and prion protein (22), were shown to be released in exosomes. A more recent study revealed that exosomes suppress cellular senescence by excreting harmful cytoplasmic DNA (23). This evidence suggests that exosomal release can attenuate cellular stress and maintain cellular homeostasis in its producing cells by exporting various unnecessary or harmful materials.

We recently reported the presence of adiponectin protein not only on the cell surface, but also in the intracellular endosomal space of in vivo aortic endothelial cells (24). Importantly, several GPI-anchored proteins have been shown to be internalized in the cells, accumulate in multivesicular bodies (MVBs), and be secreted as exosomal cargo $(22,25-27)$. T-cadherin has been shown to exist in human plasma and in ultracentrifugation precipitate fraction from the conditioned media of endothelial cells (28). Based on these observations, we hypothesized that adiponectin could enter through the endosomal route by binding to T-cadherin, a GPI-anchored protein, and exert organ protection through T-cadherin-mediated exosome modulation.

Our study establishes a potentially novel role for the adiponectin/T-cadherin system in the regulation of exosome biogenesis through adiponectin binding to T-cadherin both in vitro and in vivo. Exosomes containing adiponectin and T-cadherin are released in response to adiponectin, from cells expressing T-cadherin. Adiponectin stimulates exosome biogenesis and exosomal release of ceramides, accompanying the reduction of cellular ceramides both in vitro and in vivo. This effect should play an important role in adiponectin/T-cadherin-mediated organ protection.

\section{Results}

Intraluminal accumulation of adiponectin in vesicles of MVBs in T-cadherin-expressing endothelial cells. We initially examined T-cadherin-mediated adiponectin accumulation in endothelial F2 cells. Adiponectin accumulated in endothelial F2 cells following treatment with WT mouse serum, but not with adiponectin-knockout (AKO) mouse serum (Figure 1A). The accumulation was markedly reduced by $\mathrm{T}$-cadherin knockdown, and increased by stable overexpression of T-cadherin (Figure $1 \mathrm{~A})$. T-cadherin overexpression mainly enhanced accumulation of high- and middle-molecular-weight adiponectin, which are physiological multimers of adiponectin in blood (Figure 1A). Adiponectin also increased cellular T-cadherin protein levels (Figure 1A), as has been observed in WT mouse tissues versus AKO mouse tissues (7-9). Endothelial F2 cells stably expressing T-cadherin (F2T cells) were generated to obtain a distinct T-cadherin expression level, similar to the aorta. Equal adiponectin accumulation was observed in F2T cells and aorta (Supplemental Figure 1A; supplemental material available online with this article; https://doi.org/10.1172/jci.insight.99680DS1).

Confocal immunofluorescence microscopy demonstrated colocalization of adiponectin with T-cadherin in intracellular punctate structures (Figure 1, B and C). As expected, the majority of adiponectin colocalized with EEA1, a marker of early endosomes, and CD63, a marker of MVBs (Figure 1C), but hardly colocalized with KDEL, a marker of the endoplasmic reticulum, or ATP synthase subunit IF1 (ATPsF1), a marker of mitochondria (Supplemental Figure 1B). Immunoelectron microscopy using the pre-embedding immunoperoxidase technique demonstrated that immunoreactive adiponectin was mostly present in exosomes in MVBs of F2T cells cultured with WT mouse serum (Figure 1D, arrowhead), along with endocytic vesicles (Supplemental Figure 1C, arrowhead). However, virtually no immunoreactive signal was detected in other organelles or cytoplasm (Figure 1D and Supplemental Figure 1C). Gold nanoparticle-labeled immunoelectron microscopy analysis further demonstrated abundant accumulation of adiponectin in exosomes generated inside the MVBs (Figure 1E). Because our initial analysis of the ultrastructural localization of adiponectin in vivo in aortic endothelial cells had failed to discriminate localized endosomes (24), we investigated the localization of adiponectin in aortas again using an improved immunoelectron microscopic technique. This technical improvement facilitated the detection of many immunoreactive signals of adiponectin within endothelial cells (Figure 1, F-M). Indeed, substantial adiponectin signals were observed beneath the surface, as well as on the surface of endothelial cells (Figure 1F). Those signals were in endosomes in higher magnification, which might include MVBs, although intraluminal vesicles (ILVs) were not clearly identifiable (Figure 1, G and H). In other spots, adiponectin signals were observed as aggregates in lower magnification (Figure 1I). In higher magnification, such signals were on intraluminal structures within a visible limiting membrane, showing identifiable MVBs having many ILVs (Figure 1, J and K). 
A
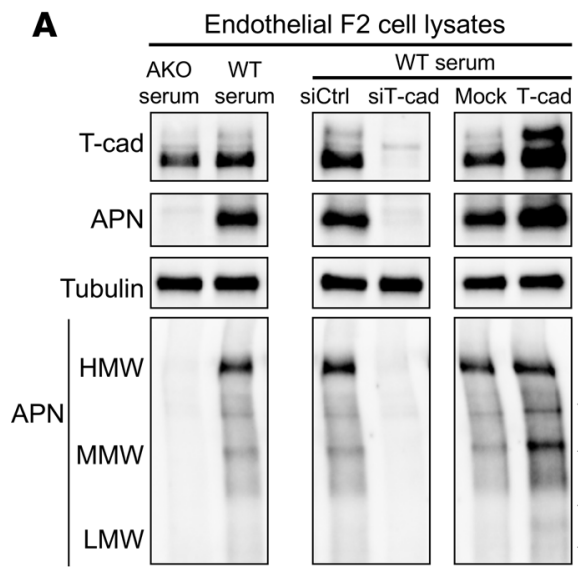

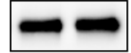

B
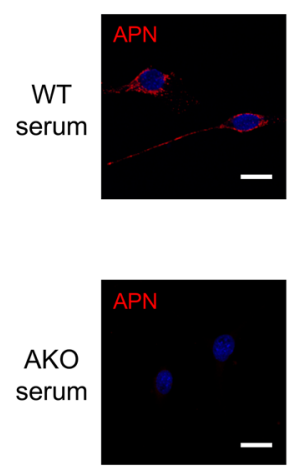

D
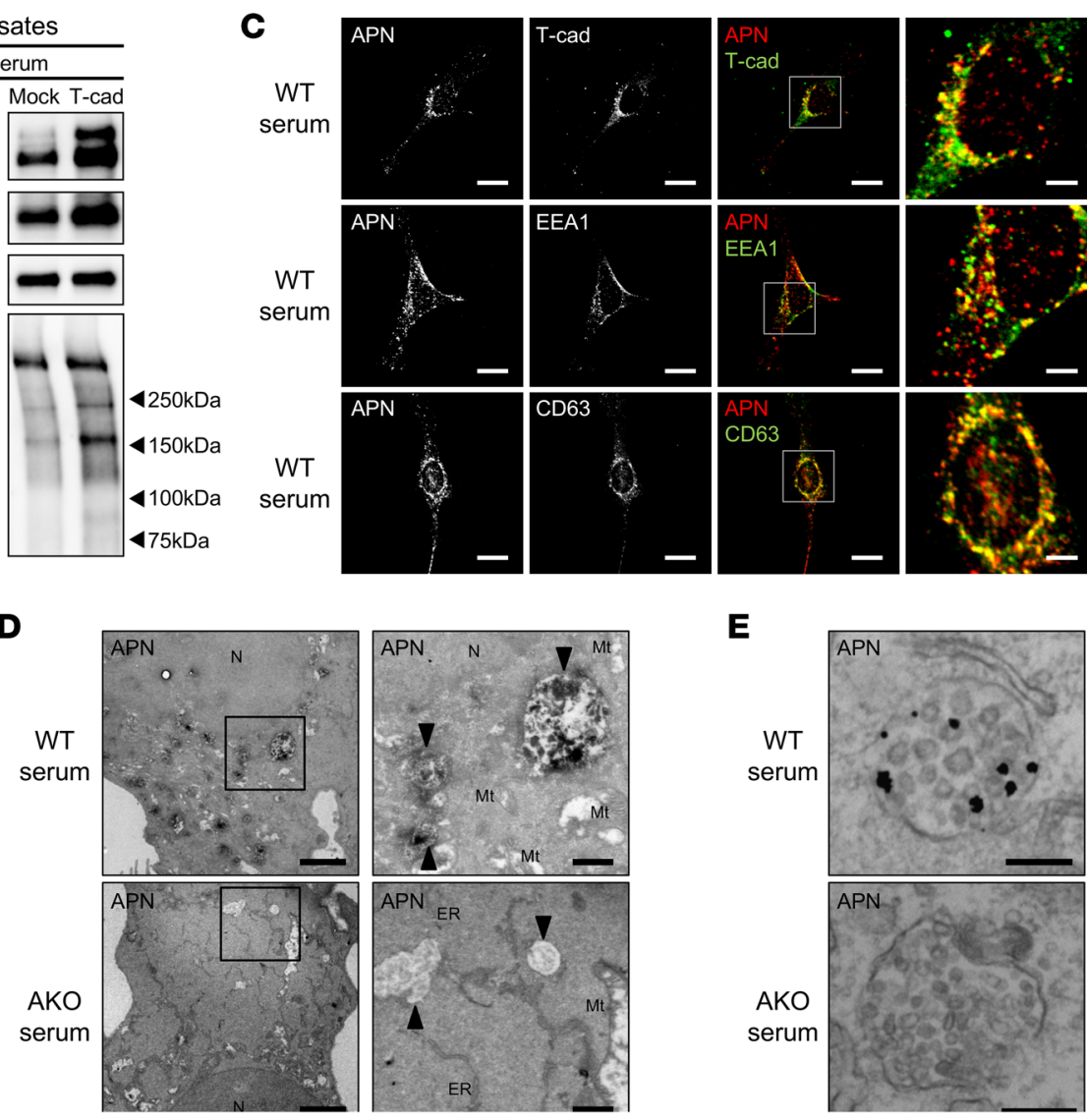

E
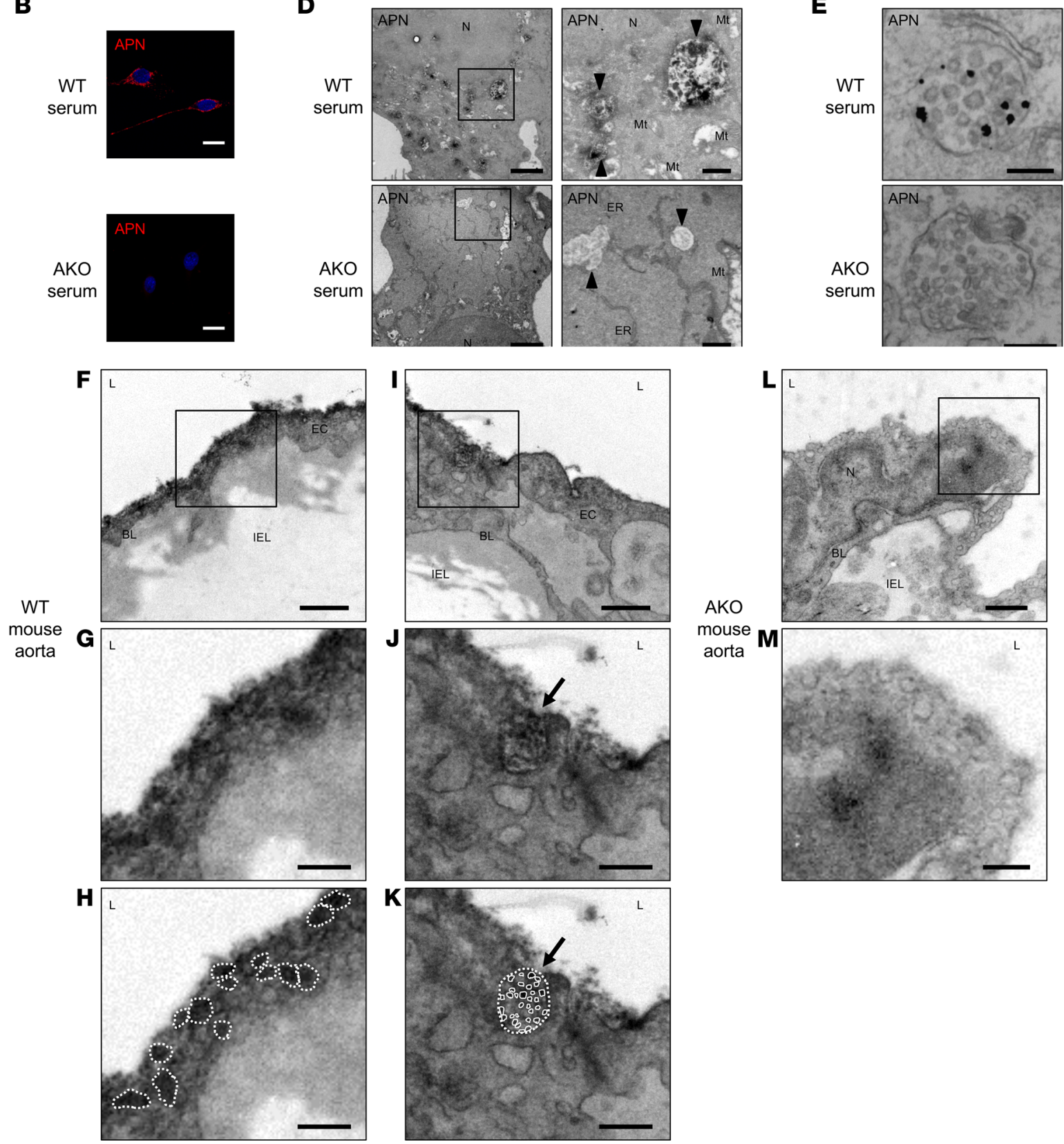

mouse $\mathbf{M}$
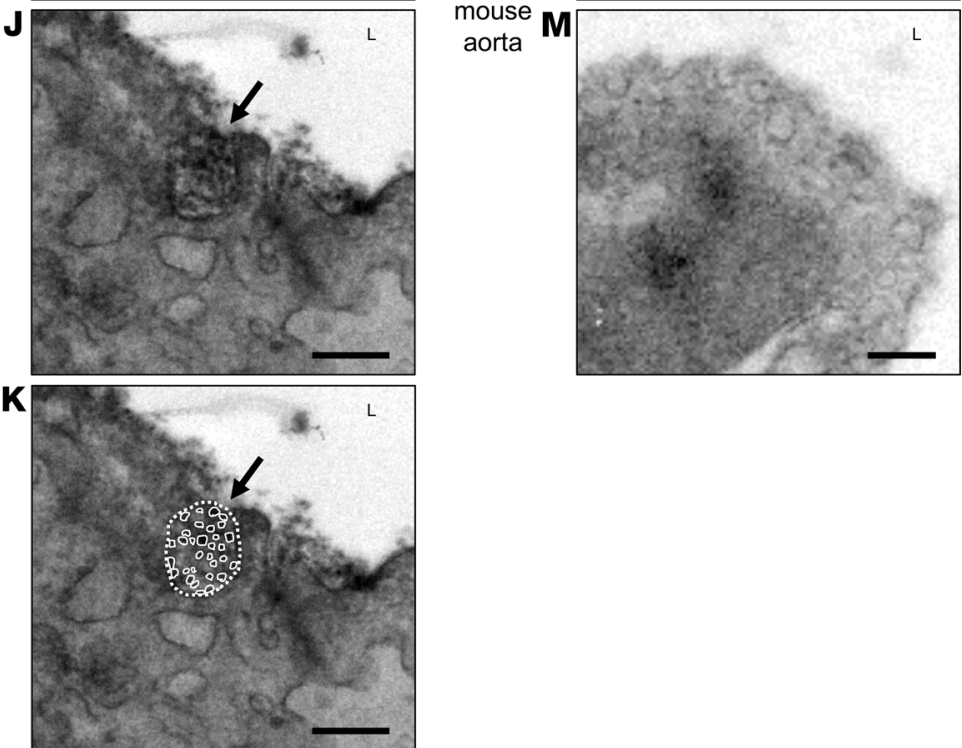
Figure 1. Intraluminal accumulation of adiponectin in vesicles of multivesicular bodies (MVBs) in T-cadherin-expressing endothelial cells. (A) Western blots of total cell lysates. F2 cells with T-cadherin depletion (by RNAi) or stably overexpressed T-cadherin (F2T cells) were treated with medium containing 5\% adiponectin-knockout (AKO) mouse serum, or WT mouse serum. APN, adiponectin; T-cad, T-cadherin; HMW, high molecular weight; MMW, middle molecular weight; LMW, low molecular weight. Representative results of 5 experiments with similar findings. (B and C) Confocal immunofluorescence micrographs of F2T cells. Cells cultured with AKO mouse serum or WT mouse serum were stained with anti-adiponectin, anti-T-cadherin, anti-EEA1 (early endosome), or anti-CD63 (MVB) antibodies. Cell nuclei were counterstained with DAPI. Scale bars: $20 \mu \mathrm{m}$. Higher magnifications of the boxed areas are shown in the right panels. Scale bars: $5 \mu \mathrm{m}$. (D) Immunoelectron micrographs using the pre-embedding immunoperoxidase technique for adiponectin in F2T cells cultured with WT mouse serum or AKO mouse serum. Right panels are higher magnifications of the regions outlined in the left panels. Scale bars: $5 \mu \mathrm{m}$ (left panels) and $1 \mu \mathrm{m}$ (right panels). Arrowhead, MVB; N, nucleus; Mt, mitochondria; ER, endoplasmic reticulum. (E) Immunoelectron micrographs using the pre-embedding immunogold labeling technique for adiponectin in F2T cells cultured with WT mouse serum or AKO mouse serum. Scale bar: $0.5 \mu \mathrm{m}$. (F-M) Immunoelectron micrographs using the pre-embedding immunoperoxidase technique for adiponectin in aorta of male WT mouse or AKO mouse. $\mathbf{G}, \mathbf{J}$, and $\mathbf{M}$ are higher magnifications of the regions outlined in $\mathbf{F}, \mathbf{I}$, and $\mathbf{L}$, respectively. (H) Endosomes in panel $\mathbf{G}$ are outlined for easier identification. (K) A limiting membrane and intraluminal structures in panel J are outlined for easier identification. Scale bars: $500 \mathrm{~nm}(\mathbf{F}, \mathbf{I}$, and $\mathbf{L})$ and 200 nm (G, H, J, K, and M). Arrow, MVB; N, nucleus; L, lumen; BL, basal lamina; IEL, internal elastic lamina.

Such signals were not observed in AKO mouse samples (Figure 1, L and M). These results were consistent with observations in cultured endothelial cells (Figure 1, D and E). Collectively, these results show that the adiponectin/T-cadherin complex enters early endosomes from the cell surface and ultimately accumulates in exosomes generated inside the MVBs.

Adiponectin is resecreted as exosomal cargo with T-cadherin. Next, we analyzed exosomes isolated from the conditioned medium of endothelial F2T cells. Following floating of exosomes, obtained by differential ultracentrifugation (UC), on discontinuous density gradients for further separation, adiponectin and T-cadherin were cofractionated with exosomal markers, such as $\mathrm{CD} 63$, milk fat globule-epidermal growth factor 8 (MFG-E8), and syntenin (Figure 2A). Immunoelectron microscopy demonstrated the presence of adiponectin in exosomes derived from culture medium of F2T cells (Figure 2B and Supplemental Figure 2). Importantly, in the presence of adiponectin (incubation with WT serum), exosomal adiponectin almost completely disappeared by knockdown and was increased by overexpression of T-cadherin (Figure 2C), resembling the pattern of cellular accumulation (Figure 1A), ruling out the possibility of nonspecific sticking of adiponectin to the exosome surface. Reflecting the T-cadherin dependence, accumulation of high-molecular-weight adiponectin, which is the physiologically active form of adiponectin, was noted in exosomes (Figure 2C), also similar to cellular accumulation (Figure 1A).

The presence of adiponectin- and T-cadherin-containing exosomes was also detected in WT mouse serum by discontinuous density gradient UC following purification by a phosphatidylserine affinity resin, as described in Methods (Figure 2D). Taken together, the results indicate that adiponectin enters the endosomal route to MVBs by binding to cell-surface T-cadherin and is secreted as exosomal cargo with T-cadherin both in vitro and in vivo.

Adiponectin affects systemic exosome levels in vivo in mice. Both adiponectin and T-cadherin widely and abundantly exist in tissues. Thus, we evaluated the in vivo effects of adiponectin on plasma exosome level. We developed a simple method that involved exosome isolation from a relatively small amount of plasma $(15 \mu \mathrm{l})$, based on polymer-based purification and a standard differential UC technique (see Methods), as also validated in a recent study (29). Using this technique, we analyzed exosome levels in mouse plasma. The plasma exosome levels of MFG-E8 and syntenin in both adiponectin heterozygous and null mutant mice were significantly lower than in WT littermate mice (Figure 3A). Furthermore, plasma exosome levels of MFG-E8 and syntenin were significantly lower in both T-cadherin heterozygous and null mutant mice than in WT littermate mice (Figure 3B). We also examined the effect of excess adiponectin on exosome production in vivo. In these experiments, adenovirus expressing adiponectin (Ad-APN) or $\beta$-galactosidase (Ad- $\beta \mathrm{Gal}$ ) was injected into WT mice and the levels of adiponectin were measured at day 4 after injection. Ad-APN increased plasma adiponectin to about $400 \mu \mathrm{g} / \mathrm{ml}$, which accompanied a 2- to 3-fold increase in plasma exosome levels of MFG-E8 and syntenin (Figure 3C).

In our plasma exosome preparations, the levels of syntenin and MFG-E8 correlated strongly in the mouse groups mentioned above (Supplemental Figure 3). A recent proteomic study recognized syntenin as a specific and common marker for all exosomes of endosomal origin (30), in addition to its functional role in exosome biogenesis (31). MFG-E8 is a phosphatidylserine binding protein (32) and binds to the exosome surface (33). Taken together, these observations suggest that the levels of these 2 markers with different routes of sorting in exosome pellets reflect plasma exosome levels. Collectively, these results demonstrate that adiponectin affects plasma exosome levels in a dose-dependent and T-cadherin-dependent manner in vivo. 
A

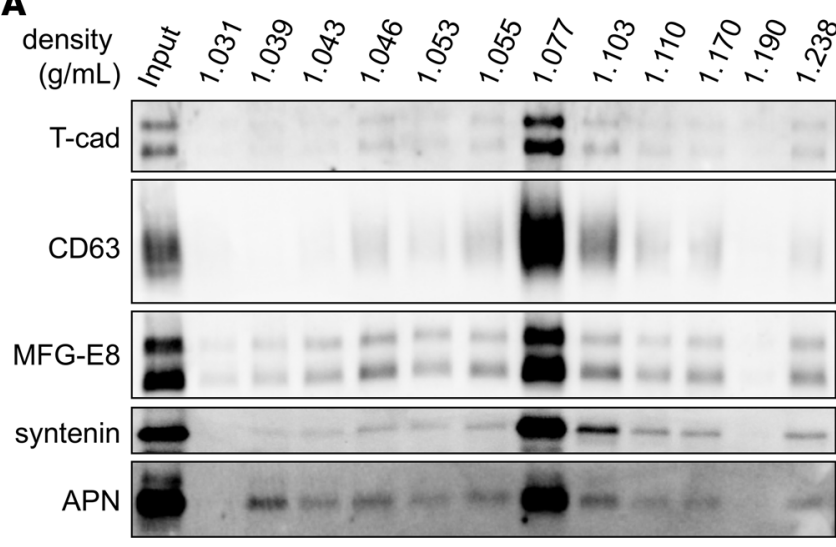

C

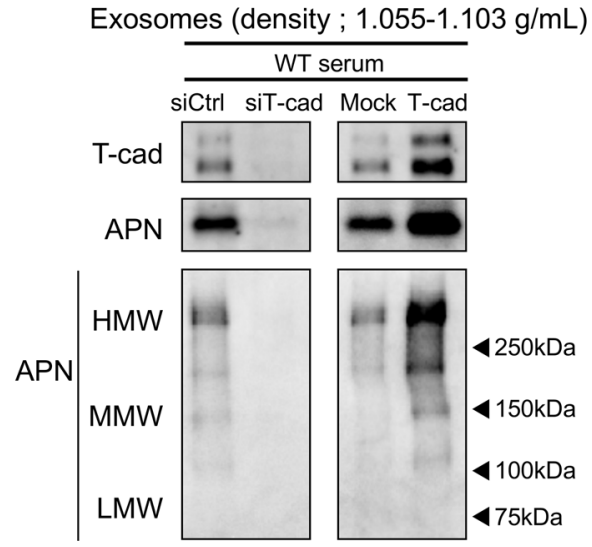

B
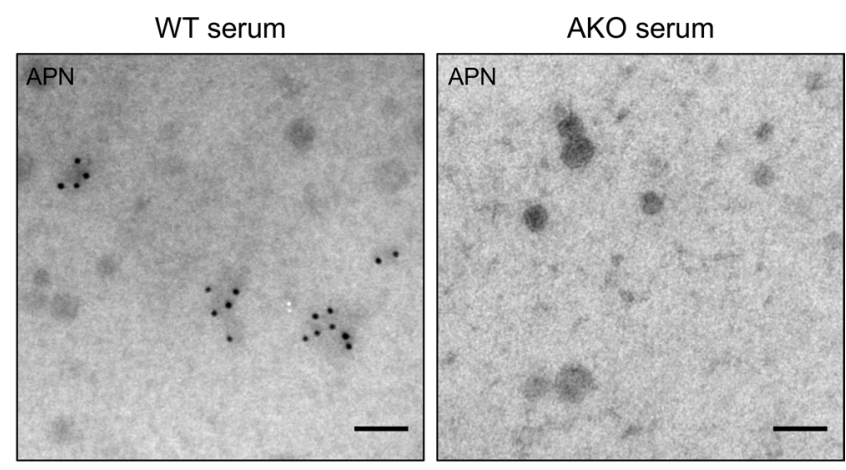

D

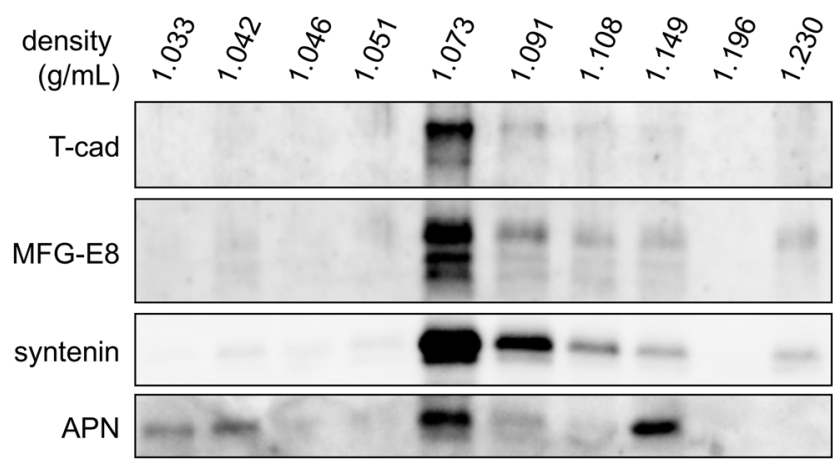

Figure 2. Adiponectin is resecreted as exosomal cargo with T-cadherin. (A) Flotation of adiponectin (APN) together with T-cadherin (T-cad) in exosome fractionation by density gradient ultracentrifugation (UC). F2T cells were cultured with $5 \%$ exosome-depleted WT mouse serum. Exosomes were isolated from the conditioned medium by differential UC and then further separated by density gradient UC. Representative results of 3 experiments with similar findings. (B) Immunoelectron micrographs of exosomes derived from F2T cells, labeled with anti-APN antibody and visualized by 10-nm-gold-conjugated secondary antibody. Cells were cultured with WT mouse serum or AKO mouse serum, and exosomes were isolated by differential UC from the conditioned medium. Scale bars: $100 \mathrm{~nm}$. (C) Western blots of exosomes, illustrating the T-cadherin-dependent APN accumulation in exosomes derived from F2 cells. Exosomes were isolated from the conditioned media of F2 cells cultured in Figure 1A. After fractionation by density gradient UC, fractions corresponding to densities of 1.055 to $1.103 \mathrm{~g} / \mathrm{ml}$ were pooled. Representative results of 5 experiments with similar findings. (D) Serum exosome fractionation by density gradient UC. Exosomes isolated from WT mouse serum by a phosphatidylserine affinity resin were further fractionated by density gradient UC. Representative results of 2 experiments with similar findings.

Adiponectin increases exosome production from T-cadherin-expressing cells. Next, we investigated the effect of adiponectin on exosome production in vitro (Figure 4 and Supplemental Figure 4). The levels of exosome markers, such as MFG-E8 and syntenin, were slightly but significantly increased, in parallel with T-cadherin when F2T cells were treated with growth medium containing 5\% WT mouse serum (final adiponectin concentration was approximately $1 \mu \mathrm{g} / \mathrm{ml}$ ), not with AKO mouse serum (Supplemental Figure 4A). To use adiponectin with active physiological properties in in vitro experiments, serum collected from AKO mice treated with Ad-APN or Ad- $\beta$ Gal was mixed to obtain the concentrations of adiponectin indicated in the figures, as described in Methods. Strikingly, at a physiological level (10 $\mu \mathrm{g} /$ $\mathrm{ml}$ ), adiponectin markedly increased exosomal T-cadherin and markers of exosomes, such as ALIX, heat shock protein 70 (Hsp70), CD63, MFG-E8, and syntenin (Figure 4A). Of note, adiponectin linearly and dose-dependently increased the amounts of exosomal T-cadherin and exosomal markers (Supplemental Figure 4B), whereas treatment with adiponectin at $3 \mu \mathrm{g} / \mathrm{ml}$ increased cellular adiponectin and T-cadherin protein to plateau levels (Supplemental Figure 4C). We also assessed the time course of adiponectin's effect on exosome production. Exosome production showed accelerated increase with cell growth, and adiponectin stimulated it gradually and chronically, not transiently (Supplemental Figure $4 \mathrm{D})$. The adiponectin-mediated increase in exosome production was also confirmed by other methods 
A

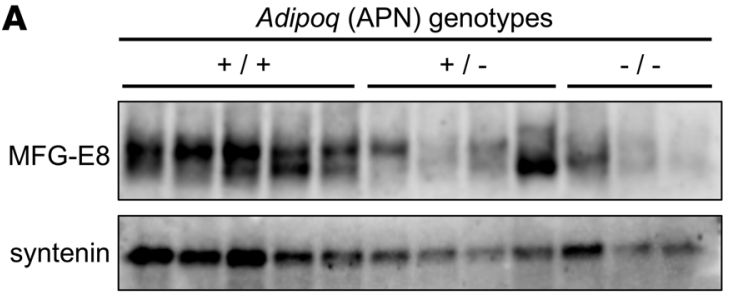

B

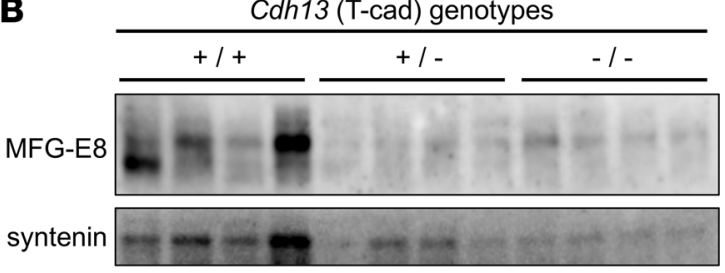

C

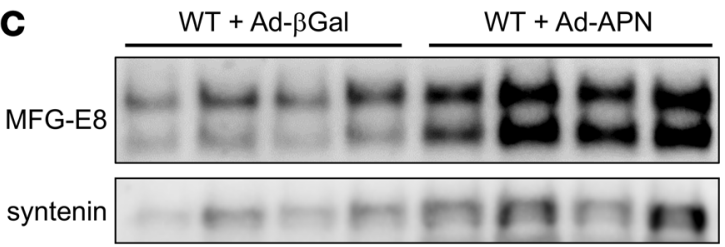

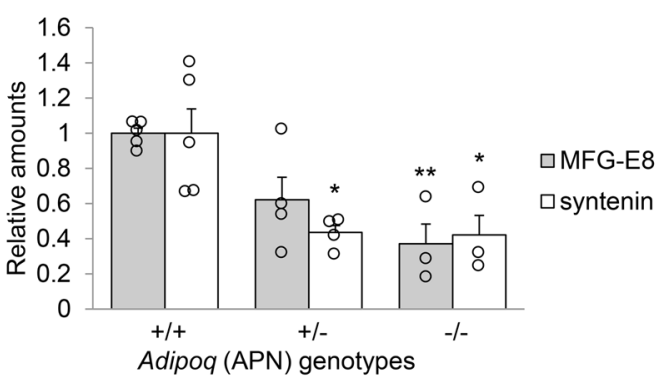
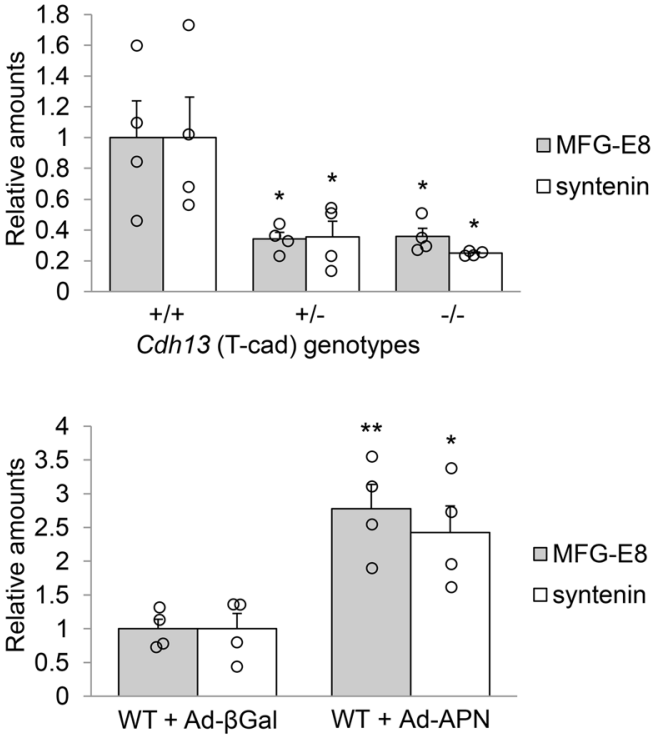

Figure 3. Adiponectin (APN) affects systemic exosome level in vivo in mouse. (A-C, left panels) Western blots of MFG-E8 and syntenin associated with exosome pellets isolated from plasma of (A) Adipoq (APN) null, heterozygous, and WT littermates; (B) Cdh13 (T-cadherin) null, heterozygous, and WT littermates; and (C) WT mice 4 days after injection of adenovirus expressing APN (Ad-APN) or $\beta$-galactosidase (Ad- $\beta$ Gal). (A and B, right panels) Exosomal MFG-E8 and syntenin levels from Western blots. ${ }^{*} P<0.05,{ }^{*} P<0.01$ versus littermate WT mice (1-way ANOVA with Tukey's post hoc test). (C, right panel) Exosomal MFG-E8 and syntenin levels from Western blot. ${ }^{*} P<0.05,{ }^{*} P<0.01$ versus WT + Ad- $\beta$ Gal (unpaired $t$ test). Data are the mean \pm SEM. Plasma APN concentrations were as follows: (A) WT $15.0 \pm 1.7 \mu \mathrm{g} / \mathrm{ml}$, heterozygous $5.0 \pm 0.5 \mu \mathrm{g} / \mathrm{ml}$, homozygous $0.0 \pm 0.0 \mu \mathrm{g} / \mathrm{ml}$; (B) WT $16.3 \pm 0.6 \mu \mathrm{g} / \mathrm{ml}$ heterozygous $28.4 \pm 2.5 \mu \mathrm{g} / \mathrm{ml}$, homozygous $44.0 \pm 2.1 \mu \mathrm{g} / \mathrm{ml}$; (C) Ad-APN $391.4 \pm 62.4 \mu \mathrm{g} / \mathrm{ml}, \mathrm{Ad}-\beta \mathrm{Gal} 21.4 \pm 2.2 \mu \mathrm{g} / \mathrm{ml}$.

(Figure 4, B and C). Acetylcholinesterase is reported to be an enzyme that is specifically directed to exosomes, and its activity reflects exosome yields (34). Adiponectin treatment significantly increased exosome-associated acetylcholinesterase activity (Figure 4B). Furthermore, nanoparticle tracking analysis showed that adiponectin significantly increased the number of particles with sizes that matched those of exosomes (Figure 4C).

To specify the effect of adiponectin, other than unknown exosome-stimulating factors possibly contained in serum, we purified adiponectin from mouse serum using T-cadherin-conjugated resin (35). Such purified protein consisted of high- and middle-molecular-weight multimers of adiponectin, even in Coomassie brilliant blue staining and in Western blotting (Figure 4D). This was consistent with the observation that T-cadherin binds only high- and middle-molecular-weight forms of adiponectin (36), even from whole serum. Such purified high-molecular-weight forms of adiponectin stimulated exosome production comparably to that of adiponectin-containing serum from Ad-APN mice (Figure 4E), indicating that exosome stimulation is mediated by high- and middle-molecular-weight adiponectin.

Adiponectin enhances exosome biogenesis. We next investigated whether adiponectin indeed enhances intracellular exosome biogenesis, by measuring the rate of de novo synthesis of exosomes in MVBs (Figure 5). To keep de novo-synthesized exosomes in MVBs, Rab7 (37) and Rab27a (38) were knocked down to inhibit fusion of MVBs/lysosomes and exosome secretion, respectively (Figure 5A). Double knockdown of these proteins caused MVBs to enlarge (Figure 5B), similarly to previous reports $(37,38)$. Newly 
A

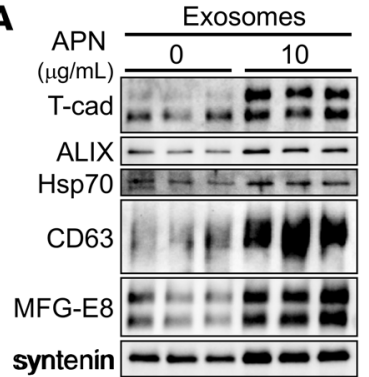

B

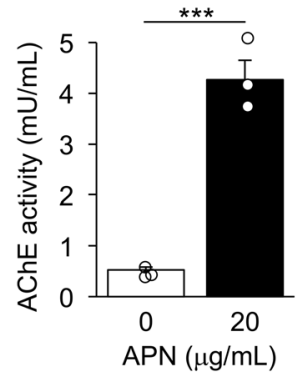

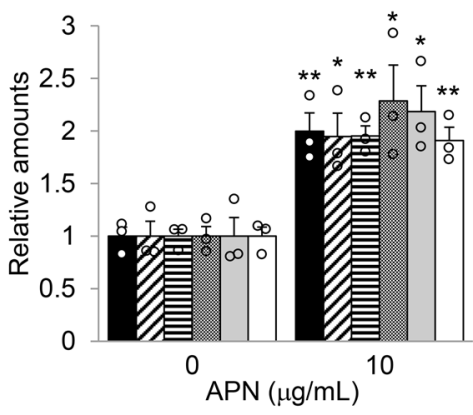

C

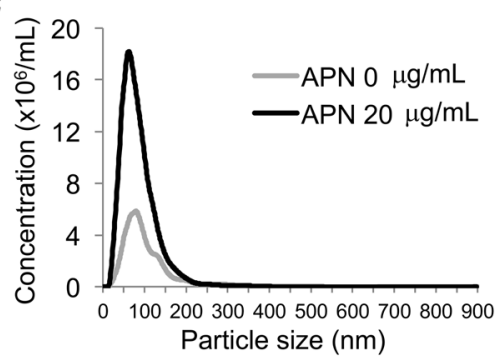

- T-cad

$\triangle A L I X$

घHsp70

금 $\mathrm{CD} 63$

口MFG-E8

$\square$ syntenin

D Purified APN

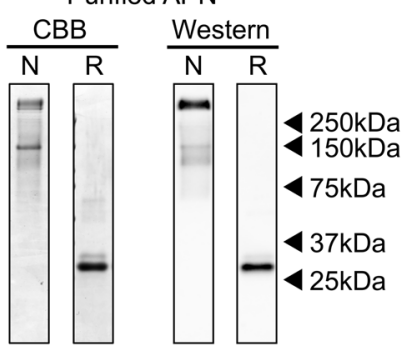

E

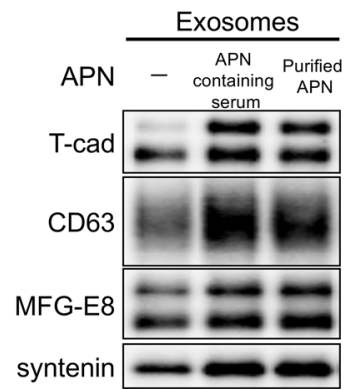

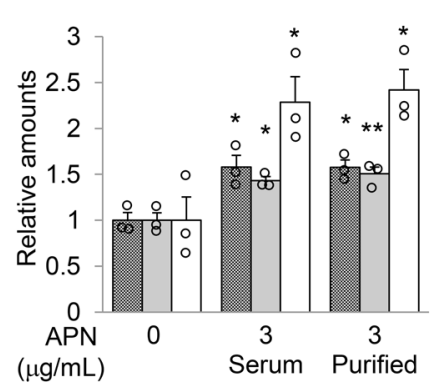

$(\mu \mathrm{g} / \mathrm{mL})$

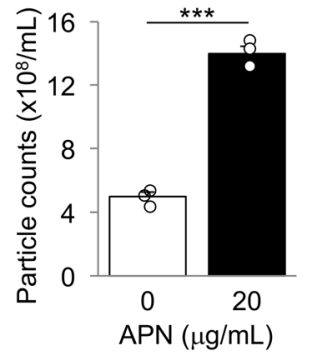

CD63

口MFG-E8

$\square$ syntenin

Figure 4. Adiponectin increases exosome production from T-cadherin-expressing cells. (A-C) Adiponectin (APN) enhanced exosome yields from F2T cells. Serum collected from APN-knockout (AKO) mice treated with adenovirus expressing APN (Ad-APN) or $\beta$-galactosidase (Ad- $\beta$ Cal) was mixed to obtain the indicated concentrations of APN. F2T cells were treated with the indicated APN concentrations, and exosomes isolated from conditioned media by differential ultracentrifugation were analyzed by Western blotting (A), acetylcholinesterase (AChE) activity (B), or nanoparticle tracking analysis (C). $n=3$ for each experiment. (A) APN increased exosomal T-cadherin (T-cad) and exosomal cargos tested. T-cad (105 and $130 \mathrm{kDa}$ ), ALIX (95 kDa), Hsp70 (70 kDa), CD63 (broad 50-100 kDa), MFG-E8 (45 and $55 \mathrm{kDa}$ ), and syntenin (32 kDa) bands were quantified, the results of which are shown in the right panel. Representative results of 5 experiments with similar findings. ${ }^{*} P<0.05$, ${ }^{* *} P<0.01$ versus $0 \mu \mathrm{g} / \mathrm{ml}$ APN (unpaired $t$ test). (B) Enzymatic quantification of AChE activities associated with exosome pellets. ${ }^{* *} P<0.001$ (unpaired $t$ test). (C) APN increased the number of exosome-sized particles. The mean particle concentration $(n=3)$ is plotted against the particle size (left panel), with total particle counts (right panel). ${ }^{* * *} P<0.001$ (unpaired $t$ test). (D) Purified APN analyzed by Coomassie brilliant blue (CBB) stain and Western blot. APN was purified from Ad-APN-injected mouse serum using T-cadherin-conjugated resin. $\mathrm{N}$, nonheating and nonreducing conditions; $\mathrm{R}$, heating and reducing conditions. (E) Comparison of the effects of APN-containing serum and T-cadherin resin-purified APN on exosome production. APN concentrations were adjusted to $3 \mu \mathrm{g} / \mathrm{ml}$. $n=3$. Representative results of 3 experiments with similar findings. ${ }^{*} P<0.05$, ${ }^{* *} P<0.01$ versus $0 \mu \mathrm{g} / \mathrm{ml}$ APN. There was no statistically significant difference between APN-containing serum and purified APN (1-way ANOVA with Tukey's post hoc test). Data are the mean \pm SEM.

synthesized exosomes in MVBs during 30 minutes of incubation were labeled by biotinylated anti-CD63 antibody uptake into MVBs (Figure 5A). Immunoelectron microscopy analysis using the pre-embedding immunoperoxidase technique demonstrated that adiponectin significantly increased the 3,3'-diaminobenzidine tetrahydrochloride-stained (DAB-stained) area in the MVBs (Figure 5C). These results indicate that adiponectin increases exosome production partly through enhancing exosome biogenesis in MVBs.

Previous studies established that exosome biogenesis is affected by the intracellular calcium chelator BAPTA-AM or siRNA knockdown of ALIX, syntenin, CD63, CD81, CD82, neutral sphingomyelinase, or sphingosine 1-phosphate receptor $(39,40)$. Those treatments reproducibly decreased basal yields of exosomes from F2T cells (Supplemental Figure 5). However, the fold increases of exosome yield by adiponectin were not significantly affected by such treatments (Supplemental Figure 5).

The effect of adiponectin on exosome production is dependent on T-cadherin, but not AdipoRs. We also investigated whether adiponectin-stimulated exosome production is dependent on T-cadherin. Adiponectin 
A

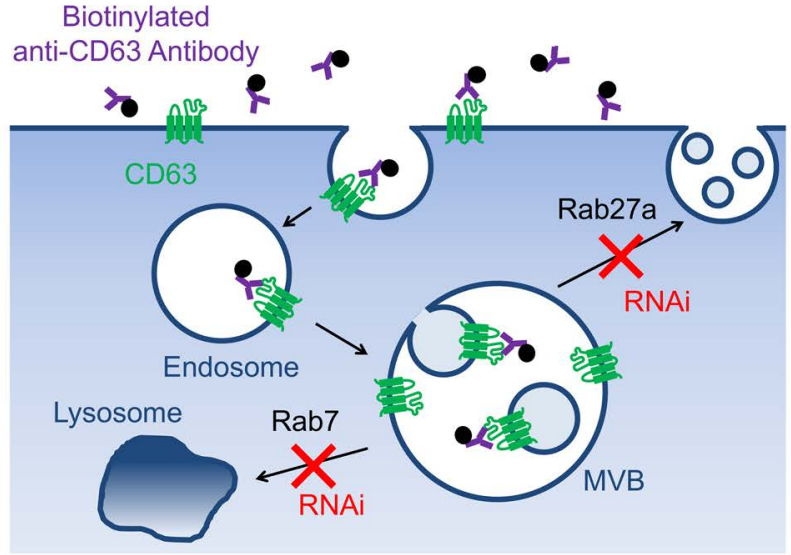

C
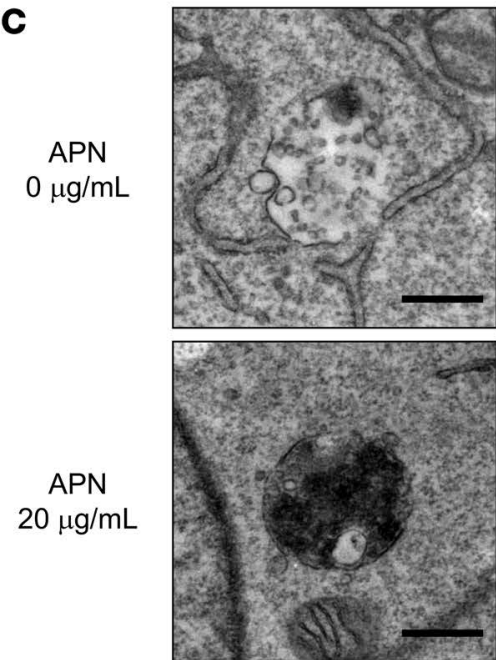
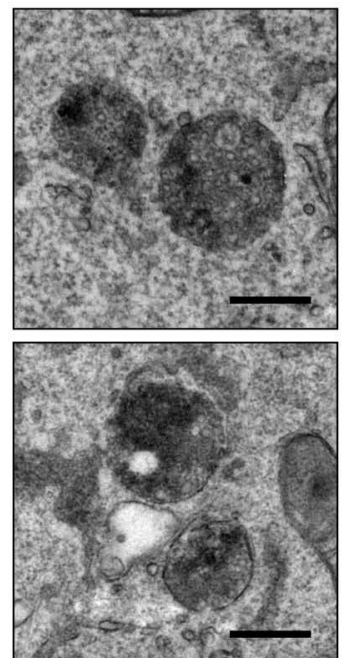

B
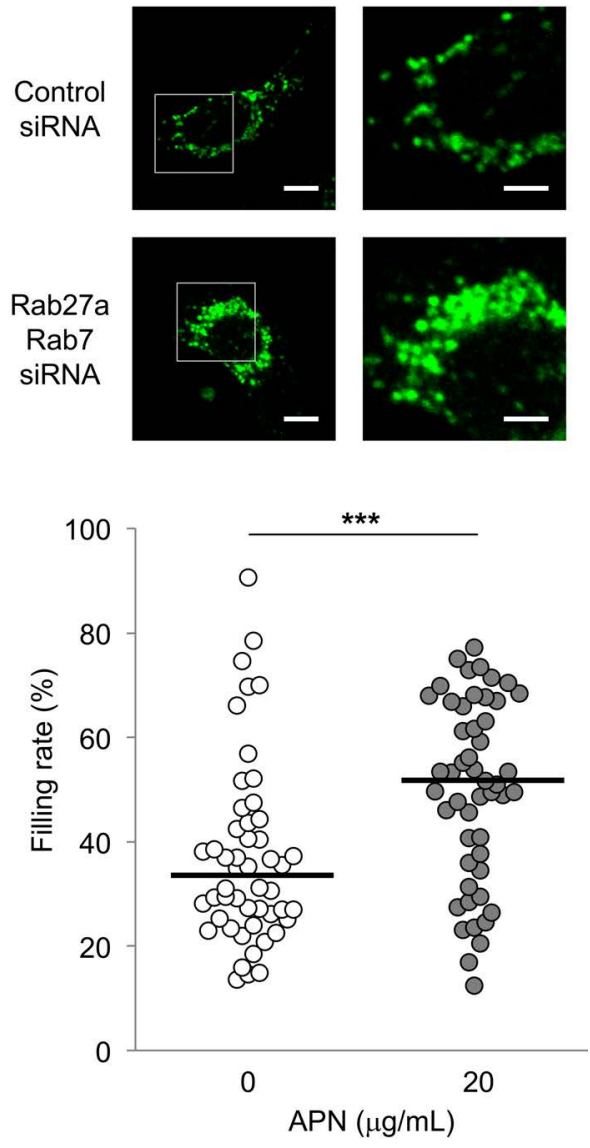

Figure 5. Adiponectin enhances exosome biogenesis. (A) Overview of the experiment for assessing the rate of de novo synthesis of exosomes. (B) Confocal immunofluorescence micrographs of F2T cells transfected with Rab7 and Rab27a siRNAs. Cells were stained with anti-CD63 antibody. Scale bars: 10 $\mu \mathrm{m}$. Higher magnifications of the boxed areas are shown in the right panels. Scale bars: $5 \mu \mathrm{m}$. (C, left panels) Representative immunoelectron micrographs using the pre-embedding immunoperoxidase technique in F2T cells with Rab7 and Rab27a double-depletion. Cells were cultured with 0 or $20 \mu \mathrm{g} /$ $\mathrm{ml}$ adiponectin (APN) and incubated with biotinylated anti-CD63 antibody for 30 minutes at $37^{\circ} \mathrm{C}$. Newly synthesized exosomes in multivesicular bodies (MVBs) were visualized by HRP-conjugated streptavidin with 3,3'-diaminobenzidine tetrahydrochloride (DAB). Scale bars: $0.5 \mu \mathrm{m}$. (C, right panel) For each DAB-stained MVB, the filling rate was calculated by dividing the stained area in the MVB by the MVB total area. Fifty MVBs for each group were tested. In the graph, horizontal bars represent the medians. ${ }^{* *} P<0.001$ (Mann-Whitney $U$ test).

significantly enhanced exosome production in F2T cells (Figure 6A). Such an effect was markedly reduced in the parental F2 cells (F2 siCtrl) and abolished by T-cadherin knockdown (F2 siT-cad), compared with F2T cells (Figure 6A). The effect of adiponectin on exosome production was also seen in other T-cadherin-expressing cell types including primary human umbilical vein endothelial cells (HUVECs) (Supplemental Figure 6A) and $\mathrm{C} 2 \mathrm{C} 12$ differentiated myotubes (Supplemental Figure 6B), but not in HepG2 hepatocytes with scarce T-cadherin expression (Supplemental Figure 6C).

We also examined the possible significance of other candidate adiponectin receptors, AdipoR1 and AdipoR2, on this effect. Knockdown of AdipoR1 or AdipoR2 had little effect on adiponectin accumulation and cellular T-cadherin protein in F2T cells (Supplemental Figure 6, D and E). More importantly, knockdown of AdipoR1 or AdipoR2 did not affect adiponectin-stimulated exosome production (Figure $6 \mathrm{~B}$ ), in contrast to T-cadherin (Figure 6A). We also carefully tested if an increase in one AdipoR by knockdown might be compensated by the other. However, there was no such compensation observed, at least in their mRNA expression levels (Supplemental Figure 6D). Indeed, adiponectin still enhanced exosome production in F2T cells even when both AdipoR1 and AdipoR2 were knocked down (Supplemental Figure 6, D and F). Furthermore, dominant-negative AMPK did not affect the adiponectin-stimulated exosome production, although it significantly decreased the phosphorylation level of AMPK in these cells 
A

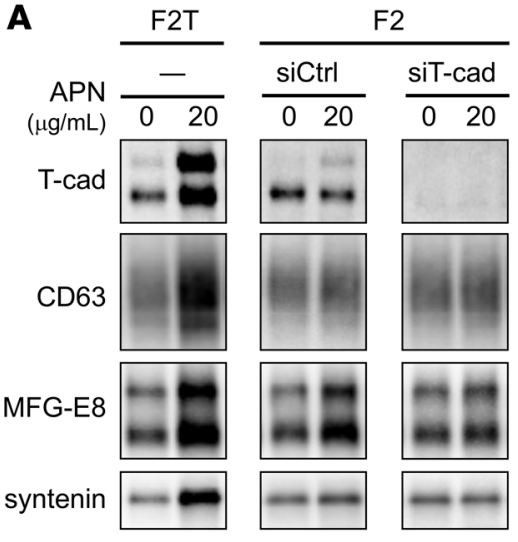

B

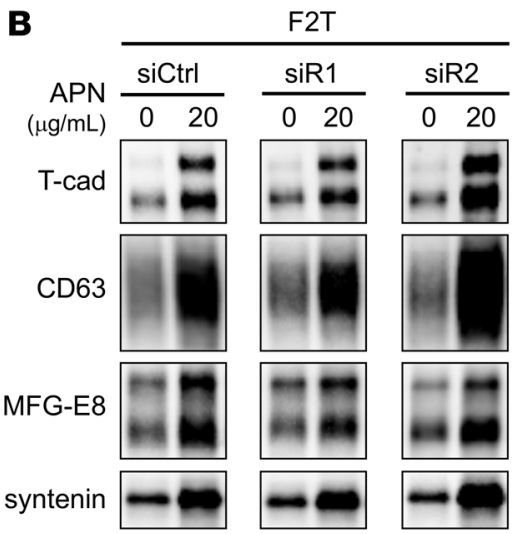

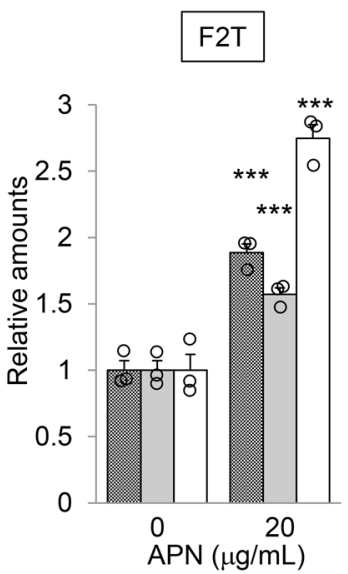
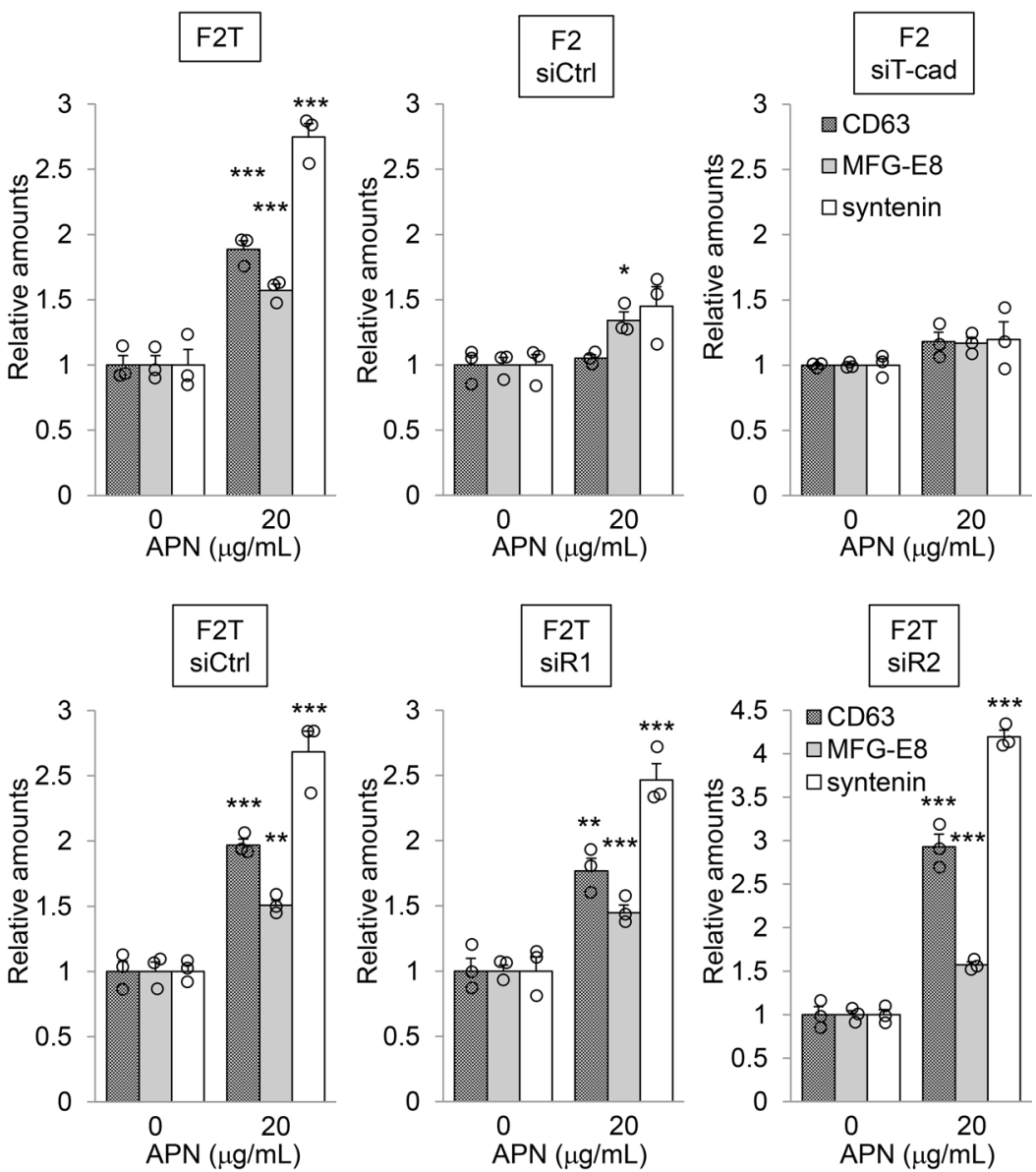

Figure 6. The effect of adiponectin on exosome production is dependent on T-cadherin, but not AdipoRs. (A) T-cadherin-dependent stimulation of exosome production by adiponectin (APN). F2T cells, parental F2 cells transfected with control siRNA (siCtrl), or T-cadherin siRNA (siT-cad) were treated with 0 or $20 \mu \mathrm{g} / \mathrm{ml}$ APN, and exosomal cargo levels were analyzed in exosome pellets by Western blotting. $n=3$. Representative results of 3 experiments with similar findings. (B) Effect of AdipoRs on exosome stimulation by APN. F2T cells transfected with control siRNA (siCtrl), AdipoR1 siRNA (siR1), or AdipoR2 siRNA (siR2) were treated with 0 or $20 \mu \mathrm{g} / \mathrm{ml} \mathrm{APN}$, and exosomal cargo levels were analyzed in exosome pellets by Western blotting. $n=3$. Representative results of 2 experiments with similar findings. Data are the mean $\pm \mathrm{SEM} .{ }^{*} P<0.05,{ }^{* *} P<0.01,{ }^{* *} P<0.001$ versus $0 \mu \mathrm{g} / \mathrm{ml}$ APN (unpaired $t$ test).

(Supplemental Figure 6, G and H). These results clearly indicate that T-cadherin, but not AdipoRs, mediates adiponectin-stimulated exosome production.

Adiponectin decreases cellular ceramides through T-cadherin-mediated exosomal release. Exosomes are enriched in ceramides, which can be exported by exosome secretion $(41,42)$. Firstly, we measured total ceramides in exosomes and cells by enzymatic derivatization and labeling of ceramide to ceramide1-phosphate using $\left[\gamma_{-1}{ }^{32} \mathrm{P}\right]$ ATP (Figure 7A). The amounts of ceramides in exosomes produced during 2 days of culture were at comparable levels to those in cells. Treatment with native adiponectin significantly increased ceramide levels in exosomes secreted from F2T cells (Figure 7A). Such ceramide release in exosomes accompanied a significant decrease of ceramide levels in F2T cells, to similar extents (Figure 7A). Next, we measured absolute amounts of ceramide species in exosomes and cells using mass spectrometry, and again observed that ceramide increases in exosomes accompanied ceramide reductions in cells by adiponectin treatment (Figure 7, B and C). Ceramides in secreted exosomes and their producing F2T endothelial cells were mainly composed of saturated ceramides, and this ceramide composition was quite similar between exosomes and cells (Figure 7, B and C). Such adiponectin effects of increasing exosomal ceramides and decreasing cellular ceramides were abolished by T-cadherin knockdown in F2T cells (Figure 7, D and E). We measured ceramidase activity (Supplemental Figure 7A), and found that adiponectin did not affect ceramidase activity at various pHs in F2T cells (Supplemental Figure 7B). Furthermore, mass spectrometry determination of sphingosine level, the enzymatic product of ceramidases in cells, showed significant reduction by adiponectin treatment (Supplemental Figure 7C), which coincides with 
A

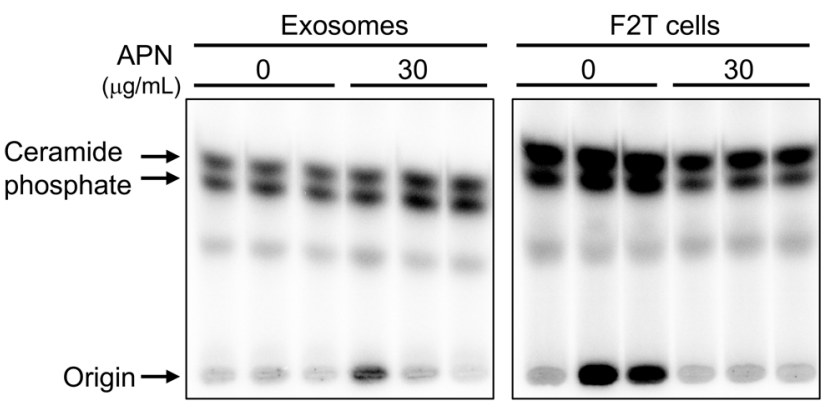

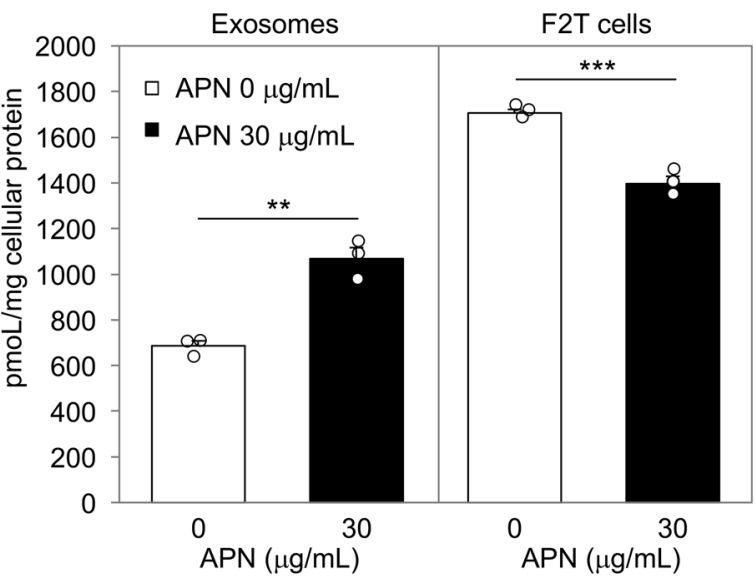

C

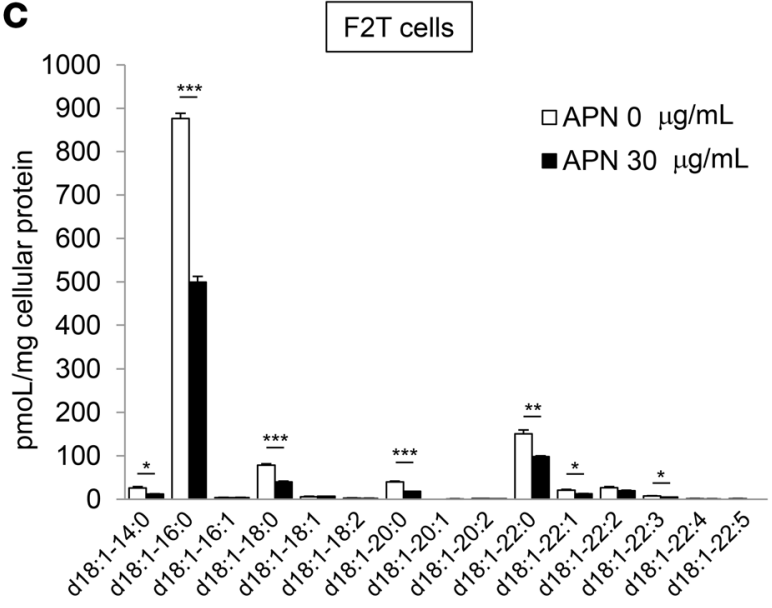

D
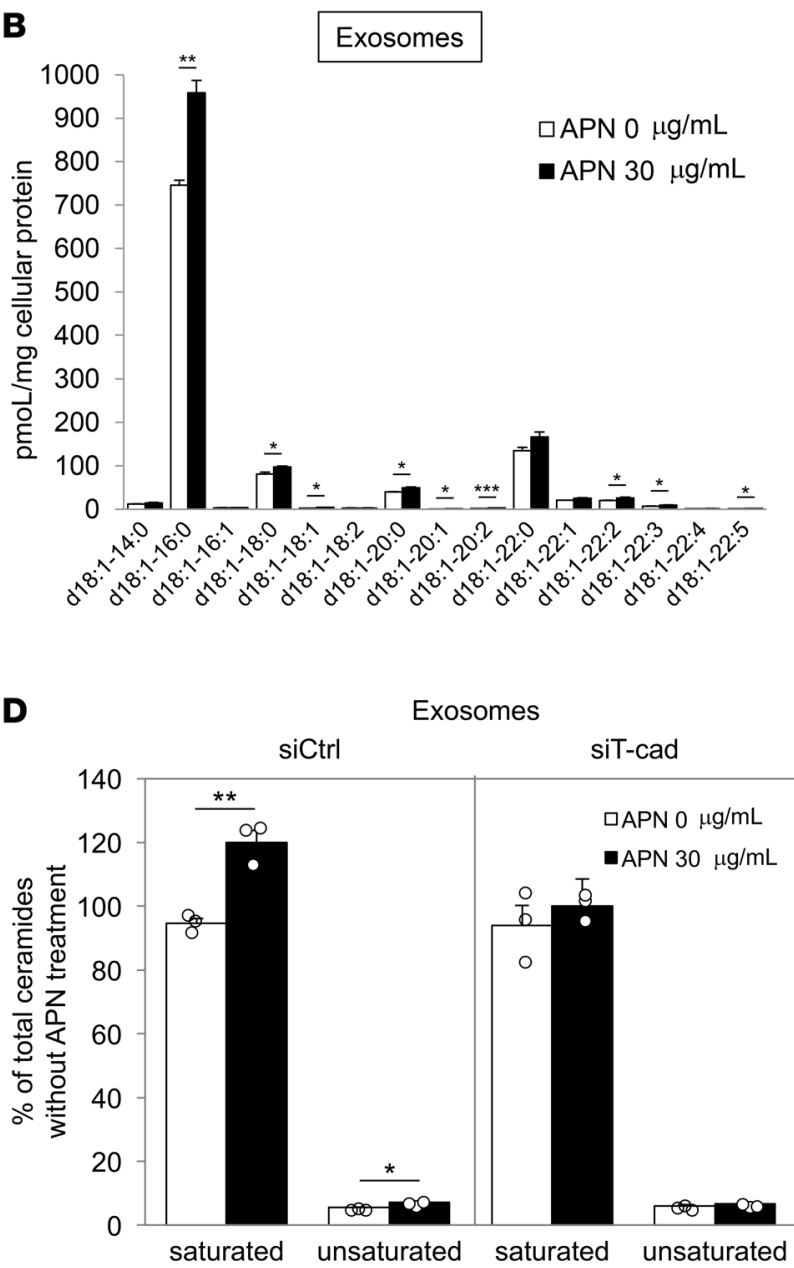

$\mathbf{E}$

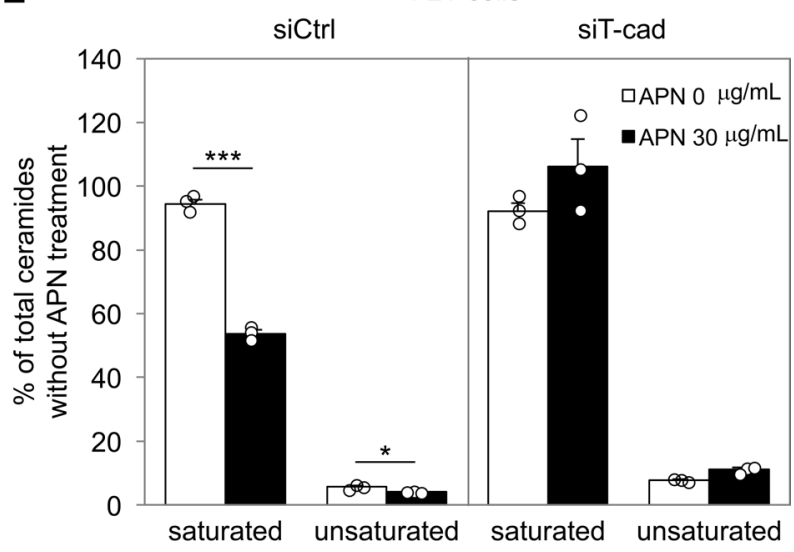

Figure 7. Adiponectin decreases cellular ceramides through T-cadherin-mediated exosomal release. (A) Total ceramide contents enzymatically determined in exosomes and cells. F2T cells were treated with 0 or $30 \mu \mathrm{g} / \mathrm{ml}$ adiponectin (APN). [ $\left.{ }^{32} \mathrm{P}\right]$ Ceramide phosphates derived from ceramides by diacylglycerol kinase using $\left[\gamma^{-32}\right.$ P]ATP were separated on thin-layer chromatography in the left panel, and quantified in the right panel. $n=3$. (B) Ceramide species contents determined with mass spectrometry in exosomes derived from F2T cells treated with 0 or $30 \mu \mathrm{g} / \mathrm{ml}$ APN. $n=3$. (C) Ceramide species contents determined with mass spectrometry in F2T cells treated with 0 or $30 \mu \mathrm{g} / \mathrm{ml}$ APN. $n=3$. (D) Effect of APN on ceramide levels in exosomes derived from F2T cells transfected with control siRNA (siCtrl) or T-cadherin siRNA (siT-cad). Each ceramide level is shown as a percentage of the total ceramides in exosomes derived from F2T cells without APN treatment. $n=3$. (E) Effect of APN on cellular ceramide levels in F2T cells transfected with control siRNA (siCtrl) or T-cadherin siRNA (siT-cad). Each ceramide level is shown as a percentage of the total ceramides in F2T cells without APN treatment. $n=3$. Data are the mean $\pm \mathrm{SEM} .{ }^{*} P<0.05,{ }^{* *} P<0.01,{ }^{* * *} P<0.001$ (unpaired $t$ test). 


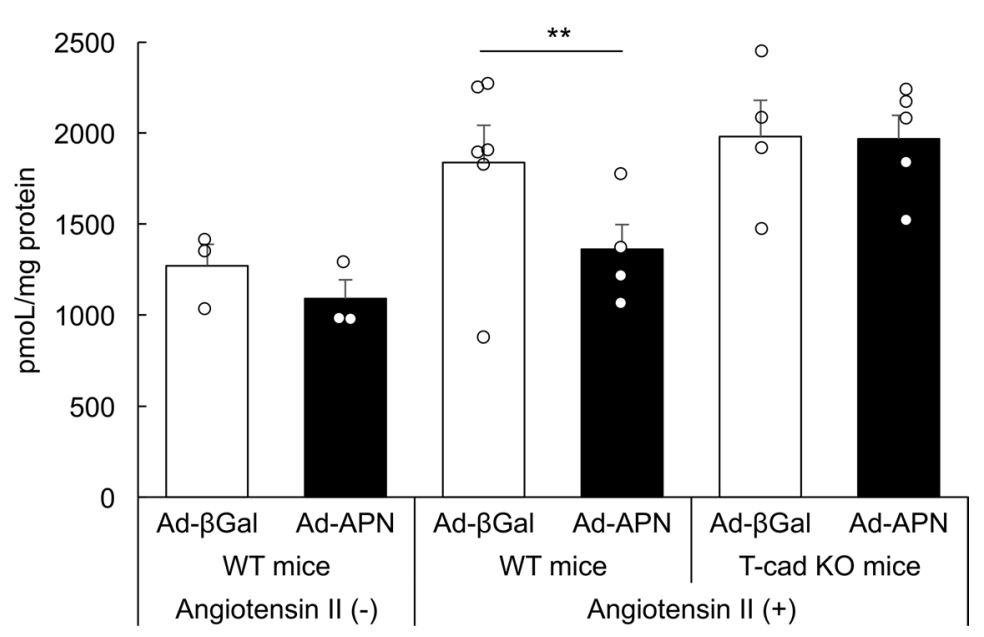

Figure 8. Adiponectin decreases ceramides in mouse aorta, dependently on T-cadherin. Aortic ceramide levels in WT mice or T-cadherin-knockout (T-cad KO) mice. WT mice or T-cad KO mice at 8 weeks of age were injected with adenovirus expressing adiponectin (Ad-APN) or $\beta$-galactosidase (Ad- $\beta$ Gal) and osmotic minipumps containing 2.4 mg/ kg/day of angiotensin II were implanted at the same time. Seven days later, aortic ceramide levels were measured. $n=3-6$ for each group. Data are the mean \pm SEM. ${ }^{*} P<0.01$ (unpaired $t$ test).

the reduction of substrate ceramide in cells by adiponectin treatment. These data demonstrate that adiponectin decreased cellular ceramides dependently on T-cadherin-mediated exosomal release, and likely not dependently on intracellular degradation by ceramidases in F2T cells.

Adiponectin decreases ceramides in mouse aorta, dependently on T-cadherin. Finally, we measured ceramide levels in aortas of WT or T-cadherin-knockout mice injected with Ad-APN or Ad- $\beta$ Gal, to investigate the significance of this ceramide-reducing effect of adiponectin in the most T-cadherin-abundant tissue in vivo. Treatment with adiponectin showed a trend toward decreasing aortic ceramide levels in WT mice (Figure 8). When mice were administered angiotensin II, ceramide levels increased, which is thought to be one of mechanisms of endothelial dysfunction in hypertension (43). Adiponectin significantly suppressed ceramide accumulation in such aortas of WT mice, but not in T-cadherin-knockout mice (Figure 8). These results are consistent with the adiponectin/T-cadherin system reducing cellular ceramides through ceramide efflux in exosomes.

\section{Discussion}

Our study establishes a potentially novel function of adiponectin, an unusually abundant adipocyte factor, which converts to an exosomal cargo, regulates exosome biogenesis by binding to T-cadherin, and decreases cellular ceramide levels by exosomal release both in vitro and in vivo. Adiponectin, together with T-cadherin, accumulates in T-cadherin-expressing cells, and is endocytosed into MVBs, where exosomes are generated. Both adiponectin and T-cadherin are released as exosomal cargo. Adiponectin has previously been suggested to exist in serum exosomes (44). Our work revealed that T-cadherin-mediated cellular processes create and secrete adiponectin-containing exosomes. Importantly, the adiponectin/T-cadherin-mediated exosome biogenesis increases ceramide efflux in exosomes, and decreases cellular ceramide levels.

Many experimental studies related to adiponectin used an E. coli-recombinant globular form of adiponectin, the presence of which has not been demonstrated in blood, or E. coli/mammalian cellproduced full-length adiponectin, which contains much less high- or middle-molecular-weight multimer. To overcome these issues, we introduced native adiponectin physiologically rich in high-molecularweight multimers contained in serum or purified from serum. We used physiological concentrations of native serum-derived adiponectin on cells expressing physiological levels of T-cadherin, which allowed the identification of this role of adiponectin in the regulation of exosome biogenesis through binding to T-cadherin.

The effect of adiponectin on exosome production did not seem to simply stimulate previously known mechanisms, such as ESCRT or tetraspanin. Interestingly, higher-order oligomerization of protein with membrane anchoring was shown to be sufficient for protein sorting into the exosome (45). Furthermore, clustering of the extremely abundant membrane protein syndecan facilitated the stimulation of total exosome production in a cell culture model, with the association of syntenin (31). It is considered that our native adiponectin, with a multimeric structure, may cause higher-order clustering of T-cadherin, a membrane-anchored protein. Adiponectin abundantly circulates at 2 to $20 \mu \mathrm{g} / \mathrm{ml}$ in 
blood, and T-cadherin protein also exists abundantly. The amount of T-cadherin protein is more than 2 orders of magnitude higher than other hormone and cytokine receptors, such as insulin receptor and TNF- $\alpha$ receptor in aortic endothelial cells in vivo (preliminary data not shown). Therefore, it can be speculated that the abundance of adiponectin and T-cadherin under physiological conditions, and the multimeric nature of adiponectin, drive exosome biogenesis in T-cadherin-expressing cells by stimulating clustering of T-cadherin. Because T-cadherin exhibits a wide tissue distribution, including heart, vascular endothelium, and skeletal muscles, the adiponectin/T-cadherin system may ultimately affect systemic exosome levels in the blood.

Growing evidence has suggested exosomes are a molecular signaling package for some specific target cells $(17,18)$. Exosomes are also suggested to dispose unnecessary or harmful materials from their producing cells (20-23). Several studies have implied that ceramide is one of the enriched cargos in exosomes $(41,42)$. In our study, adiponectin enhanced exosome biogenesis, increased ceramide efflux in exosomes, and comparably decreased cellular ceramide species dependently on T-cadherin, without affecting ceramidase activity in endothelial cells. It has been thought that sphingolipid homeostasis is maintained by the balance between intracellular synthesis and degradation. This study may be the first to demonstrate that ceramide efflux in exosomes contributes to cellular ceramide homeostasis. Furthermore, adiponectin increased serum exosome levels and significantly suppressed ceramide accumulation in mouse aortas under angiotensin II infusion. As intracellular accumulation of ceramides, especially saturated ceramides, is involved in several pathogeneses such as insulin resistance and endothelial dysfunction (46-51), adiponectin/T-cadherin-mediated decrease of cellular ceramide via exosome biogenesis and secretion should be protective against metabolic disorders and organ damage. Also, adiponectin may contribute to the maintenance of cellular homeostasis by enhancing excretion of various unnecessary or harmful materials besides ceramides, a hypothesis that is now under investigation. One of the major target cell types of exosomes is thought to be macrophages, specific scavenger cells (52-54). It has been reported that exosomes are internalized more efficiently by macrophages via phagocytosis, than by non-phagocytic cells (52). This finding is supported by the fact that phosphatidylserine, which is recognized by macrophages in phagocytosis of apoptotic cells (32), is exposed on the surface of exosomal membranes. Knockdown of Tim4, a key molecule in phagocytosis (55), attenuated exosome internalization in macrophages (52). Additionally, more recent studies demonstrated that most exosomes are quickly taken up by macrophages in the liver after intravenous injection in mice $(53,54)$. These data suggest that ceramides and other harmful materials released in exosomes may be targeted to and degraded by macrophages, whose degradative activity, including that towards ceramides, is very high.

Ceramide-lowering activity is thought to be an important mechanism of adiponectin to combat metabolic diseases and to protect against organ damage $(56,57)$. It was reported that adiponectin decreased cellular ceramides by activating ceramidase activities of AdipoRs (56). This finding was further supported by inducible overexpression of AdipoRs in the liver, accompanying improved glucose metabolism (57). Crystal structures of sphingosine in the AdipoR2 binding pocket have provided additional support for the ceramidase concept in adiponectin action (58). Our study indicates that T-cadherin, in response to multimeric adiponectin, functions to increase exosome biogenesis and also decrease cellular ceramides in endothelial cells expressing T-cadherin. Importantly, no obvious accumulation of multimeric adiponectin was observed in liver, where little or no T-cadherin exists, in sharp contrast to aortic endothelium, heart, and muscle, where T-cadherin is abundantly expressed (9). T-cadherin may regulate cellular ceramide content in response to high-molecular-weight multimeric adiponectin, in a tissue-dependent manner as an additional mechanism for the adiponectin/AdipoR axis.

It has also been suggested that exosomes, released into the extracellular environment, protect organs by modulating the immune function of the recipient cells (59), or by stimulating the repair ability of the recipient cells (60-62). Thus, adiponectin-induced exosome release may modulate the function of recipient cells, protectively.

Taken together, the results suggest that stimulation of exosome production by adiponectin may partly explain the adiponectin-related organ protection through the GPI-anchored protein T-cadherin, which may lead to creating novel therapeutic strategies for organ protection. Importantly, this is the first demonstration to our knowledge that a circulating factor stimulates exosome biogenesis that affects ceramide metabolism and the circulating pool of exosomes. 


\section{Methods}

Antibodies. The following primary antibodies were used: goat polyclonal anti-adiponectin (AF1119, R\&D Systems); rabbit polyclonal anti-adiponectin (PA1-054, Thermo Fisher Scientific); goat polyclonal antiT-cadherin (AF3264, R\&D Systems); rat monoclonal anti-mouse CD63 (clone R5G2, MBL); mouse monoclonal anti-human CD63 (clone H5C6, BD); rabbit polyclonal anti-syntenin (ab19903, Abcam); goat polyclonal anti-MFG-E8 (AF2805, R\&D Systems); rabbit monoclonal anti- $\alpha$-tubulin (11H10, Cell Signaling Technology); mouse monoclonal anti-ALIX (clone 3A9, Santa Cruz Biotechnology); rabbit polyclonal anti-Hsp70 (EXOAB-Hsp70A-1, System Biosciences); rabbit polyclonal anti-EEA1 (clone H-300, Santa Cruz Biotechnology); mouse monoclonal anti-KDEL (clone 10C3, Stressgen); mouse monoclonal antiATP synthase subunit IF1 (A-21355, Thermo Fisher Scientific); rabbit polyclonal anti-AMPK $\alpha$ (2532, Cell Signaling Technology); and rabbit polyclonal anti-phospho-AMPK $\alpha$ (2531, Cell Signaling Technology). The following secondary antibodies were used: horseradish peroxidase-conjugated (HRP-conjugated) rabbit anti-goat IgG and rabbit anti-mouse IgG antibodies (Invitrogen); HRP-conjugated goat anti-rat IgG antibody (GE Healthcare); HRP-conjugated goat anti-rabbit IgG antibody (Millipore); Alexa Fluor-conjugated secondary antibodies (Life Technologies); biotin-conjugated rabbit anti-goat IgG antibody (Life Technologies); nanogold-conjugated rabbit anti-goat IgG secondary antibody (Nanoprobes); goat anti-rat IgG and rabbit anti-goat IgG secondary antibodies conjugated with 10-nm gold particles (Cytodiagnostics).

Animals and serum adiponectin. AKO mice and T-cadherin-knockout mice were generated previously in the C57BL/6J background $(63,64)$. Serum samples used for cell culture experiments were collected under anesthesia via the inferior vena cava from 12- to 16-week-old male WT and AKO mice. To obtain high levels of high-molecular-weight adiponectin, Ad-APN or Ad- $\beta$ Gal was purified using the Adenovirus Standard Purification Virakit (Virapur). AKO mice were injected with Ad-APN or Ad- $\beta$ Gal at $2.0 \times$ $10^{9}$ plaque-forming units/mouse via the tail vein. Blood samples were collected from the respective mice at day 4 after injection, and serum adiponectin levels were measured by ELISA (Otsuka Pharmaceutical Co.). Serum from AKO mice injected with Ad-APN was diluted with that from AKO mice injected with Ad- $\beta \mathrm{Gal}$ to obtain the indicated adiponectin concentrations. Mouse serum used for exosome isolation was depleted of exosomes by UC at maximum 100,000 $\mathrm{g}$ for 14 hours (SW55Ti rotor, Beckman Coulter). Adiponectin concentration was measured in the resultant exosome-free serum and adjusted as described above.

Adiponectin purification. Adiponectin purification was performed as reported previously (35). Briefly, serum from AKO mice treated with Ad-APN was applied onto T-cadherin-Fc conjugated with Protein G sepharose (GE healthcare). Adiponectin was eluted with 5 mM EDTA.

Cell culture. Mouse vascular endothelial F2 cells were obtained from RIKEN Cell Bank and maintained in DMEM with 10\% fetal bovine serum (FBS), $100 \mathrm{U} / \mathrm{ml}$ penicillin, and $100 \mu \mathrm{g} / \mathrm{ml}$ streptomycin. To generate F2 cells stably overexpressing T-cadherin (F2T cells), complementary DNA encoding the full-length mouse T-cadherin was subcloned into pMXs-puro, and the resultant vector was used to transfect Plat-E cells and thereby generate recombinant retroviruses. F2 cells were infected with the recombinant retroviruses and selected in growth medium containing $2 \mu \mathrm{g} / \mathrm{ml}$ puromycin. For the AMPK study, the dominantnegative pAMPK $\alpha 2 \mathrm{~K} 45 \mathrm{R}$ construct was obtained from Morris Birnbaum (University of Pennsylvania, Philadelphia, Pennsylvania, USA) (Addgene plasmid 15992) (65). For RNAi experiments, F2 cells were transfected with silencer select siRNA (Ambion) by using Lipofectamine RNAiMAX reagent (Life Technologies) according to the protocol supplied by the manufacturer. Incubation with adiponectin-containing media started 36 hours after transfection. HUVECs were obtained from Lonza and maintained in EGM-2 medium up to passage 6. C2C12 mouse myoblast cells were cultured in DMEM with 10\% FBS, 100 U/ $\mathrm{ml}$ penicillin, and $100 \mu \mathrm{g} / \mathrm{ml}$ streptomycin. Myogenic differentiation was induced by culture in collagen Icoated plates with DMEM supplemented with $2 \%$ horse serum, and incubation in adiponectin-containing medium commenced on day 4 . HepG2 cells were cultured in DMEM supplemented with 10\% FBS, and used for experiments.

Exosome isolation. Exosome isolation from the cell culture supernatant was performed essentially as described previously (66), with some modifications. Briefly, after overnight serum starvation, the cells were cultured with medium containing 5\% exosome-depleted mouse serum for 48 hours (F2 cells, HepG2 cells), 24 hours (HUVECs), or 12 hours ( $\mathrm{C} 2 \mathrm{C} 12$ myotubes). Then, the conditioned medium was collected and centrifuged at $800 \mathrm{~g}$ for 10 minutes to deplete floating cells, and at 10,000 $\mathrm{g}$ for 30 minutes to remove cell debris. For exosome isolation, the supernatant was ultracentrifuged at average 110,000 $\mathrm{g}$ for 2 hours, followed by a washing step of the exosome pellet with Dulbecco's phosphate-buffered saline with calcium and magnesium 
[PBS(+)] at average 110,000 $g$ for 2 hours (TLA100.1 rotor, Beckman Coulter). The exosome pellets were directly solubilized in Laemmli sample buffer or resuspended in PBS(+) containing $0.05 \%$ Tween 20 . For comparative analysis, in gain- and loss-of-function studies, exosomes were collected from equivalent amounts of culture medium, conditioned by equivalent numbers of cells. Essentially none of the mouse serum treatments, overexpression, or RNAi treatment significantly affected cell viability. The density and size distribution of exosomes were analyzed using NanoSight LM10-HS apparatus and NTA2.3 software (Malvern).

Exosome fractionation. Fractionation of exosomes by density gradient UC was performed as described previously (67) with slight modifications. A discontinuous iodixanol gradient, consisting of 40\% w/v, 20\% $\mathrm{w} / \mathrm{v}, 10 \% \mathrm{w} / \mathrm{v}$, and $5 \% \mathrm{w} / \mathrm{v}$ solutions of iodixanol, was prepared by diluting a stock solution of OptiPrep $\left(60 \% \mathrm{w} / \mathrm{v}\right.$ aqueous iodixanol, Cosmo Bio) in $0.25 \mathrm{M}$ sucrose, $10 \mathrm{mM}$ Tris- $\mathrm{HCl} \mathrm{pH}$ 7.4, $1.0 \mathrm{mM} \mathrm{CaCl}_{2}$, and $0.3 \mathrm{mM} \mathrm{MgCl}_{2}$. The gradient was set up in a polyallomer tube by subsequent layering of each solution. Exosome pellets obtained by UC from conditioned medium were suspended in PBS(+) containing $0.05 \%$ Tween 20 . Serum exosomes were purified by a phosphatidylserine-affinity magnetic resin, MagCapture Exosome Isolation Kit PS (Wako Pure Chemical). Exosome suspensions were layered on top of 5\% iodixanol solution and centrifuged at maximum 100,000 $\mathrm{g}$ for 18 hours (SW41Ti rotor, Beckman Coulter). Individual fractions were collected and diluted with PBS(+) and ultracentrifuged at average 200,000 $\mathrm{g}$ for 2 hours. The pellets were directly solubilized in Laemmli sample buffer and analyzed by Western blotting.

Western blotting. Exosomes or whole-cell lysates were loaded onto 4\%-20\% gradient SDS-PAGE gels (Bio-Rad) and transferred onto nitrocellulose membranes. The membranes were blocked with PVDF Blocking Reagent for Can Get Signal (TOYOBO) and then incubated with primary antibodies using Can Get Signal Solution 1 (TOYOBO) overnight at $4^{\circ} \mathrm{C}$ and followed by incubation with secondary antibodies conjugated with HRP using Can Get Signal Solution 2 (TOYOBO) for 60 minutes at room temperature. Chemiluminescence signals developed with Chemi-Lumi One Super (Nacalai Tesque) were visualized by ChemiDoc Touch, and quantified using Image Lab software (Bio-Rad). CD63 was detected under nonreducing conditions. Separation of the multimeric complexes of adiponectin was performed under nonheating and nonreducing conditions, as described previously (2).

Immunofluorescence staining. Cells treated with WT or AKO mouse serum on coverslips were fixed with periodate-lysine-paraformaldehyde (PLP) for 30 minutes and incubated with $3 \% \mathrm{w} / \mathrm{v}$ BSA and $0.3 \% \mathrm{w} / \mathrm{v}$ Triton X-100 in Dulbecco's phosphate-buffered saline free of calcium and magnesium (PBS) for 60 minutes. Cells were then incubated with the indicated primary antibodies overnight at $4^{\circ} \mathrm{C}$ and followed by incubation with Alexa Fluor-conjugated secondary antibodies for 60 minutes at room temperature. Goat polyclonal anti-adiponectin antibody was used for double staining of adiponectin and others except that of T-cadherin, while rabbit polyclonal anti-adiponectin antibody was used for double staining of adiponectin and T-cadherin. Cell nuclei were counterstained with DAPI. Microscopy analysis was performed using an Olympus FV1000D confocal laser scanning microscope system (Olympus).

Immunoelectron microscopy. Cells were fixed with PLP for 30 minutes, rinsed with PBS and $50 \mathrm{mM}$ glycine, and permeabilized with $0.25 \% \mathrm{w} / \mathrm{v}$ saponin in $0.1 \mathrm{M}$ phosphate buffer $(\mathrm{PB})$ for 30 minutes. The permeabilized cells were incubated with $10 \% \mathrm{w} / \mathrm{v}$ BSA, $10 \% \mathrm{w} / \mathrm{v}$ rabbit serum, $0.1 \% \mathrm{w} / \mathrm{v}$ cold-water-fish gelatin, and $0.1 \% \mathrm{w} / \mathrm{v}$ saponin in $0.1 \mathrm{M} \mathrm{PB}$ for 60 minutes and incubated with goat anti-adiponectin antibody overnight at $4^{\circ} \mathrm{C}$. Dissected aortas following perfusion with $\mathrm{PBS}(+)$ were fixed with PLP overnight at $4^{\circ} \mathrm{C}$. After washing with $0.1 \mathrm{M} \mathrm{PB}$, the tissues were immersed in $6 \%$ gelatin and then $12 \%$ gelatin solution. The samples were cut into $40-\mu \mathrm{m}$-thick sections using vibrating blade microtome VT1000s (Leica). After rinsing with $0.1 \mathrm{M} \mathrm{PB}$ and $0.1 \% \mathrm{w} / \mathrm{v} \mathrm{NaBH}_{4}$, tissues were incubated with $1 \% \mathrm{w} / \mathrm{v} \mathrm{FBS}$ and $0.1 \% \mathrm{w} / \mathrm{v}$ saponin in $0.1 \mathrm{M}$ PB for 60 minutes and incubated with goat anti-adiponectin antibody overnight at $4^{\circ} \mathrm{C}$. In the preembedding immunoperoxidase technique, labeling was visualized by biotinylated anti-goat IgG antibody and avidin-biotin-HRP procedure (Vectastatin ABC kit, Vector Laboratories) with DAB (Sigma-Aldrich) according to the protocol recommended by the manufacturer. After thorough washing with $0.1 \mathrm{M} \mathrm{PB}$, the cells were refixed in $0.1 \mathrm{M} \mathrm{PB}$ containing $1 \%$ glutaraldehyde. For pre-embedding immunogold labeling, cells treated with goat anti-adiponectin antibody were incubated with nanogold-conjugated rabbit anti-goat IgG secondary antibody for 2 hours at room temperature and then refixed in $0.1 \mathrm{M}$ PB containing $1 \%$ glutaraldehyde. To amplify signals to detectable levels, gold enhancement was performed using GoldEnhance EM (Nanoprobes) according to the instructions provided by the manufacturer. Finally, samples immunostained using both methods were prepared for electron microscopy as described previously (68). Immunoelectron microscopy analysis of exosomes was performed as described previously (69) with slight modifications. 
Exosomes suspended in PBS(+) were loaded on formvar-coated grids for 60 minutes and fixed by loading directly the same volume of $4 \%$ paraformaldehyde that was used for the exosome suspension. Grids were incubated with the primary antibody overnight at $4^{\circ} \mathrm{C}$ followed by incubation with the secondary antibody conjugated with 10-nm gold particles for 2 hours at room temperature. After thorough washing, the grids were refixed in $2.5 \%$ glutaraldehyde and then stained with $4 \%$ uranyl acetate, followed by embedding in $1.8 \%$ methylcellulose and $0.4 \%$ uranyl acetate. All electron micrographs were obtained using an H-7650 transmission electron microscope (Hitachi).

Exosome biogenesis analysis. For assessing the rate of de novo synthesis of exosomes, F2T cells transfected with Rab7 and Rab27a siRNAs were incubated with biotinylated anti-CD63 antibody for 30 minutes at $37^{\circ} \mathrm{C}$. Cells were then washed with PBS and fixed with $3 \%$ glutaraldehyde in $0.1 \mathrm{M}$ PB for 60 minutes. After thorough washing, incorporated anti-CD63 antibody was visualized by HRP-conjugated streptavidin with DAB and further processed for electron microscopy as described above.

Real-time quantitative PCR. Isolation of total RNA and synthesis of CDNA were performed using SuperPrep Cell Lysis \& RT kit for qPCR (TOYOBO). Real-time quantitative PCR was performed using SYBR green. The results for each sample were normalized to the respective 36B4 mRNA levels. Primers were as follows: 36B4, 5'-GGCCAATAAGGTGCCAGCT-3' (forward) and 5'-TGATCAGCCCGAAGGAGAAG-3' (reverse); AdipoR1, 5'-AATGGGGCTCCTTCTGGTAAC-3' (forward) and 5'- GCAGACCTTATACACGAACTCC-3' (reverse); AdipoR2， 5'-GGAGTGTTCGTGGGCTTAGG-3' (forward) and 5'-GCAGCTCCGGTGATATAGAGG-3' (reverse).

Acetylcholinesterase activity. Acetylcholinesterase activity was measured as described previously (34) with slight modifications. Briefly, $25 \mu \mathrm{l}$ of the exosomes suspended in PBS(+) containing $0.05 \%$ Tween 20 was

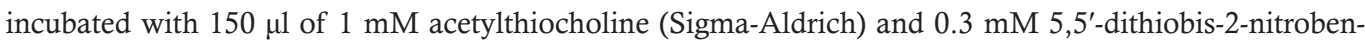
zoic acid (Sigma-Aldrich) in the same buffer. Absorbance was monitored at $405 \mathrm{~nm}$ every 60 seconds at $37^{\circ} \mathrm{C}$ with a microplate reader (SH9000ab, Hitachi), and the slope values were fitted with those of standard acetylcholinesterase from electric eel (Sigma-Aldrich).

Ceramide analysis. Cellular and exosomal lipids were extracted with a modified Brier/Dyer method. Total ceramides were enzymatically derivatized and labeled by diacylglycerol kinase using $\left[\gamma-{ }^{32} \mathrm{P}\right] \mathrm{ATP}(70)$. The reactions were extracted and separated on thin-layer chromatography, and radioactivities associated with $\left[{ }^{32} \mathrm{P}\right]$ ceramide-1-phosphates were measured by Typhoon PhosphorImager. The levels of ceramide species and sphingosine were quantified by liquid chromatography and tandem mass spectrometry using a triple-quadrupole mass spectrometer (Xevo TQ-S micro, Waters Co.) equipped with a supercritical fluid chromatography (ACQUITY UPC2, Waters Co.). Ceramide (d18:1-17:0) was used as an internal standard and values were adjusted by cell proteins.

Ceramidase activity assay. Ceramidase activity was determined using C12-NBD-ceramide as substrate with minor modifications (71). C12-NBD-ceramide dissolved in ethanol was dispersed in 2\% BSA solution. Cell pellets were suspended in $1 \mathrm{mM}$ Tris-HCl, $\mathrm{pH} 7.4$ containing protease inhibitor cocktail and disrupted by sonication. Reaction mixture composed of $20 \mu 1$ substrate solution containing $1 \mathrm{nmol} \mathrm{C12}$ NBD-ceramide, $20 \mu \mathrm{l}$ cell lysate and $10 \mu \mathrm{pH}$-buffer were incubated for 30 minutes. To achieve different $\mathrm{pH}$ conditions, $125 \mathrm{mM}$ ammonium acetate ( $\mathrm{pH}$ 4.5, 5.0), $125 \mathrm{mM}$ Bis-Tris ( $\mathrm{pH}$ 5.5, 6.0, 6.5), $25 \mathrm{mM}$ Tris ( $\mathrm{pH}$ 7.0), or $125 \mathrm{mM}$ HEPES ( $\mathrm{pH} 7.5,8.0,9.5$ ) was used as $\mathrm{pH}$-buffer. The reaction was stopped by mixing with chloroform/methanol, and lipids extracted by a modified Bligh and Dyer method was analyzed by reversephase HPLC equipped with fluorescence detector (72).

Plasma exosome analysis. Plasma was collected via the tail vein from 8-week-old male Adipoq (adiponectin) null, heterozygous, and WT littermate mice, $C d h 13$ (T-cadherin) null, heterozygous, and WT littermate mice. Plasma samples were collected under anesthesia from the inferior vena cava of 14-week-old male C57BL/6J WT mice, 4 days after injection of Ad-APN or Ad- $\beta G a l$, at $5.0 \times 10^{8}$ plaque-forming units/ mouse. Plasma was mixed with thrombin for 10 minutes at room temperature and centrifuged at $10,000 \mathrm{~g}$ for 30 minutes at $4^{\circ} \mathrm{C}$ to remove fibrin. Plasma exosomes were isolated based on polymer-based purification and standard differential UC technique. A similar combination strategy was precisely validated (29). The supernatants were incubated with ExoQuick (System Biosciences) for 30 minutes at $4^{\circ} \mathrm{C}$, and then centrifuged at $1,500 \mathrm{~g}$ for 30 minutes at $4^{\circ} \mathrm{C}$. The pellets were resuspended in PBS(+) and ultracentrifuged at average 140,000 $\mathrm{g}$ for 2 hours, followed by a washing step of the exosome pellet with PBS(+) at average $140,000 \mathrm{~g}$ for 2 hours (TLA100.1 rotor, Beckman Coulter). The obtained pellets were directly solubilized in Laemmli sample buffer and analyzed by Western blotting. 
Angiotensin II infusion. WT male mice at 8 weeks of age were injected with Ad-APN or Ad- $\beta$ Gal and osmotic minipumps (Alzet mini-osmotic pump model 2002, Durect Corp.) containing $2.4 \mathrm{mg} / \mathrm{kg} /$ day of angiotensin II (Sigma-Aldrich) dissolved in $0.01 \mathrm{M}$ acetic acid were implanted in the midscapular region. Seven days later, mice were sacrificed and aortic ceramide levels were quantified.

Statistics. Data are expressed as the mean \pm SEM. $P$ values were calculated by 2 -tailed unpaired $t$ test, Mann-Whitney $U$ test, or 1-way analysis of variance (ANOVA) with Tukey's post hoc test. Correlation between plasma exosome MFG-E8 and syntenin levels was analyzed by Pearson's correlation test.

Study approval. The experimental protocol was approved by the Ethics Review Committee for Animal Experimentation of Osaka University School of Medicine. This study also conforms to the Guide for the Care and Use of Laboratory Animals published by the US NIH.

\section{Author contributions}

YO and SK designed the research protocol, performed biochemical, cellular, and in vivo experiments, analyzed the data, and cowrote the manuscript. YK performed and analyzed confocal fluorescent microscopy and electron microscopy data. SF purified adiponectin. SF, YF, H. Nagao, SM, YT, and YN conducted parts of cellular and in vivo experiments. HT, MT, YI, TB, and EF analyzed sphingolipids by mass spectrometry. SS oversaw microscopy analyses and provided input on research design. RH oversaw exosome experiments and provided input on research design. BR provided T-cadherin-KO mice. SK and IS directed the research and cowrote the manuscript. TF, H. Nishizawa, and NM contributed to reviewing and editing the manuscript. All authors discussed and interpreted the data.

\section{Acknowledgments}

The authors thank the staff of the Center of Medical Research and Education, Graduate School of Medicine Osaka University, for the excellent technical support. We also thank the staff of the Core Instrumentation Facility, RIMD, Osaka University, for the excellent technical support. This work was supported in part by a Grant-in-Aid for Scientific Research (C) 16K09802 (to S. Kita), a Grant-in-Aid for Scientific Research (C) 16K09801 (to N. Maeda), and a Grant-in-Aid for Scientific Research (B) 15H04853 (to I. Shimomura); and grants from Takeda Science Foundation (to N. Maeda), Japan Foundation for Applied Enzymology (to Y. Fujishima), and CREST, JST (to E. Fukusaki and I. Shimomura). The funding agencies had no role in the study design, data collection and analysis, decision to publish, or preparation of the manuscript.

Address correspondence to: Shunbun Kita, Department of Metabolic Medicine, Graduate School of Medicine, Osaka University, 2-2 Suita, Osaka 565-0871, Japan. Phone: 81.6.6879.3732; Email: shunkita@endmet.med.osaka-u.ac.jp.

1. Pajvani UB, et al. Structure-function studies of the adipocyte-secreted hormone Acrp30/adiponectin. Implications fpr metabolic regulation and bioactivity. J Biol Chem. 2003;278(11):9073-9085.

2. Waki $\mathrm{H}$, et al. Impaired multimerization of human adiponectin mutants associated with diabetes. Molecular structure and multimer formation of adiponectin. J Biol Chem. 2003;278(41):40352-40363.

3. Hara K, et al. Measurement of the high-molecular weight form of adiponectin in plasma is useful for the prediction of insulin resistance and metabolic syndrome. Diabetes Care. 2006;29(6):1357-1362.

4. Inoue $\mathrm{T}$, et al. High molecular weight adiponectin as a predictor of long-term clinical outcome in patients with coronary artery disease. Am J Cardiol. 2007;100(4):569-574

5. Hirose H, Yamamoto Y, Seino-Yoshihara Y, Kawabe H, Saito I. Serum high-molecular-weight adiponectin as a marker for the evaluation and care of subjects with metabolic syndrome and related disorders. J Atheroscler Thromb. 2010;17(12):1201-1211

6. Basu R, Pajvani UB, Rizza RA, Scherer PE. Selective downregulation of the high molecular weight form of adiponectin in hyperinsulinemia and in type 2 diabetes: differential regulation from nondiabetic subjects. Diabetes. 2007;56(8):2174-2177.

7. Denzel MS, Scimia MC, Zumstein PM, Walsh K, Ruiz-Lozano P, Ranscht B. T-cadherin is critical for adiponectin-mediated cardioprotection in mice. J Clin Invest. 2010;120(12):4342-4352.

8. Parker-Duffen JL, et al. T-cadherin is essential for adiponectin-mediated revascularization. J Biol Chem. 2013;288(34):2488624897.

9. Matsuda K, et al. Positive feedback regulation between adiponectin and T-cadherin impacts adiponectin levels in tissue and plasma of male mice. Endocrinology. 2015;156(3):934-946.

10. Fujishima Y, et al. Adiponectin association with T-cadherin protects against neointima proliferation and atherosclerosis. FASEB J. 2017;31(4):1571-1583.

11. Chung CM, et al. A genome-wide association study reveals a quantitative trait locus of adiponectin on CDH13 that predicts cardiometabolic outcomes. Diabetes. 2011;60(9):2417-2423.

12. Fava C, et al. A variant upstream of the CDH13 adiponectin receptor gene and metabolic syndrome in Swedes. Am J Cardiol. 
2011;108(10):1432-1437.

13. Gao H, et al. Genetic variation in CDH13 is associated with lower plasma adiponectin levels but greater adiponectin sensitivity in East Asian populations. Diabetes. 2013;62(12):4277-4283.

14. Jee SH, et al. Adiponectin concentrations: a genome-wide association study. Am J Hum Genet. 2010;87(4):545-552.

15. Wu Y, et al. Genome-wide association study for adiponectin levels in Filipino women identifies CDH13 and a novel uncommon haplotype at KNG1-ADIPOQ. Hum Mol Genet. 2010;19(24):4955-4964.

16. Morisaki H, et al. CDH13 gene coding T-cadherin influences variations in plasma adiponectin levels in the Japanese population. Hum Mutat. 2012;33(2):402-410.

17. Couzin J. Cell biology: The ins and outs of exosomes. Science. 2005;308(5730):1862-1863.

18. Tkach M, Théry C. Communication by extracellular vesicles: Where we are and where we need to go. Cell. 2016;164(6):1226-1232.

19. Eitan E, Suire C, Zhang S, Mattson MP. Impact of lysosome status on extracellular vesicle content and release. Ageing Res Rev. 2016;32:65-74.

20. Rajendran L, et al. Alzheimer's disease beta-amyloid peptides are released in association with exosomes. Proc Natl Acad Sci USA 2006;103(30):11172-11177.

21. Saman S, et al. Exosome-associated tau is secreted in tauopathy models and is selectively phosphorylated in cerebrospinal fluid in early Alzheimer disease. J Biol Chem. 2012;287(6):3842-3849.

22. Fevrier B, et al. Cells release prions in association with exosomes. Proc Natl Acad Sci USA. 2004;101(26):9683-9688.

23. Takahashi A, et al. Exosomes maintain cellular homeostasis by excreting harmful DNA from cells. Nat Commun. 2017;8:15287.

24. Mori T, et al. Ultrastructural localization of adiponectin protein in vasculature of normal and atherosclerotic mice. Sci Rep. 2014;4:4895.

25. Clayton A, A1-Taei S, Webber J, Mason MD, Tabi Z. Cancer exosomes express CD39 and CD73, which suppress T cells through adenosine production. J Immunol. 2011;187(2):676-683.

26. Fernández-Messina L, et al. Differential mechanisms of shedding of the glycosylphosphatidylinositol (GPI)-anchored NKG2D ligands. J Biol Chem. 2010;285(12):8543-8551.

27. Grapp M, et al. Choroid plexus transcytosis and exosome shuttling deliver folate into brain parenchyma. Nat Commun. 2013;4:2123.

28. Philippova M, et al. T-cadherin is present on endothelial microparticles and is elevated in plasma in early atherosclerosis. Eur Heart J. 2011;32(6):760-771.

29. Rider MA, Hurwitz SN, Meckes DG. ExtraPEG: a polyethylene glycol-based method for enrichment of extracellular vesicles Sci Rep. 2016;6:23978.

30. Kowal J, et al. Proteomic comparison defines novel markers to characterize heterogeneous populations of extracellular vesicle subtypes. Proc Natl Acad Sci USA. 2016;113(8):E968-E977.

31. Baietti MF, et al. Syndecan-syntenin-ALIX regulates the biogenesis of exosomes. Nat Cell Biol. 2012;14(7):677-685.

32. Hanayama R, Tanaka M, Miwa K, Shinohara A, Iwamatsu A, Nagata S. Identification of a factor that links apoptotic cells to phagocytes. Nature. 2002;417(6885):182-187.

33. Miyasaka K, Hanayama R, Tanaka M, Nagata S. Expression of milk fat globule epidermal growth factor 8 in immature dendritic cells for engulfment of apoptotic cells. Eur J Immunol. 2004;34(5):1414-1422.

34. Savina A, Furlán M, Vidal M, Colombo MI. Exosome release is regulated by a calcium-dependent mechanism in K562 cells J Biol Chem. 2003;278(22):20083-20090.

35. Fukuda $\mathrm{S}$, et al. The unique prodomain of T-cadherin plays a key role in adiponectin binding with the essential extracellular cadherin repeats 1 and 2. J Biol Chem. 2017;292(19):7840-7849.

36. Hug C, Wang J, Ahmad NS, Bogan JS, Tsao TS, Lodish HF. T-cadherin is a receptor for hexameric and high-molecular-weight forms of Acrp30/adiponectin. Proc Natl Acad Sci USA. 2004;101(28):10308-10313.

37. Vanlandingham PA, Ceresa BP. Rab7 regulates late endocytic trafficking downstream of multivesicular body biogenesis and cargo sequestration. J Biol Chem. 2009;284(18):12110-12124.

38. Ostrowski M, et al. Rab27a and Rab27b control different steps of the exosome secretion pathway. Nat Cell Biol. 2010;12(1):19-30

39. Colombo M, Raposo G, Théry C. Biogenesis, secretion, and intercellular interactions of exosomes and other extracellular vesicles. Annu Rev Cell Dev Biol. 2014;30:255-289.

40. Kajimoto T, Okada T, Miya S, Zhang L, Nakamura S. Ongoing activation of sphingosine 1-phosphate receptors mediates maturation of exosomal multivesicular endosomes. Nat Commun. 2013;4:2712.

41. Trajkovic K, et al. Ceramide triggers budding of exosome vesicles into multivesicular endosomes. Science. 2008;319(5867):1244-1247.

42. Llorente A, et al. Molecular lipidomics of exosomes released by PC-3 prostate cancer cells. Biochim Biophys Acta. 2013;1831(7):1302-1309.

43. Spijkers LJ, et al. Hypertension is associated with marked alterations in sphingolipid biology: a potential role for ceramide. PLoS ONE. 2011;6(7):e21817

44. Phoonsawat W, Aoki-Yoshida A, Tsuruta T, Sonoyama K. Adiponectin is partially associated with exosomes in mouse serum. Biochem Biophys Res Commun. 2014;448(3):261-266.

45. Fang Y, Wu N, Gan X, Yan W, Morrell JC, Gould SJ. Higher-order oligomerization targets plasma membrane proteins and HIV gag to exosomes. PLoS Biol. 2007;5(6):e158.

46. Xia JY, et al. Targeted induction of ceramide degradation leads to improved systemic metabolism and reduced hepatic steatosis Cell Metab. 2015;22(2):266-278.

47. Aerts JM, et al. Pharmacological inhibition of glucosylceramide synthase enhances insulin sensitivity. Diabetes. 2007;56(5):1341-1349.

48. Holland WL, et al. Inhibition of ceramide synthesis ameliorates glucocorticoid-, saturated-fat-, and obesity-induced insulin resistance. Cell Metab. 2007;5(3):167-179.

49. Zhao H, et al. Inhibiting glycosphingolipid synthesis improves glycemic control and insulin sensitivity in animal models of type 2 diabetes. Diabetes. 2007;56(5):1210-1218 
50. Bismuth J, Lin P, Yao Q, Chen C. Ceramide: a common pathway for atherosclerosis? Atherosclerosis. 2008;196(2):497-504.

51. Edsfeldt A, et al. Sphingolipids contribute to human atherosclerotic plaque inflammation. Arterioscler Thromb Vasc Biol. 2016;36(6):1132-1140

52. Feng D, et al. Cellular internalization of exosomes occurs through phagocytosis. Traffic. 2010;11(5):675-687.

53. Imai T, et al. Macrophage-dependent clearance of systemically administered B16BL6-derived exosomes from the blood circulation in mice. J Extracell Vesicles. 2015;4:26238.

54. Charoenviriyakul C, Takahashi Y, Morishita M, Matsumoto A, Nishikawa M, Takakura Y. Cell type-specific and common characteristics of exosomes derived from mouse cell lines: Yield, physicochemical properties, and pharmacokinetics. Eur J Pharm Sci. 2017;96:316-322.

55. Miyanishi M, Tada K, Koike M, Uchiyama Y, Kitamura T, Nagata S. Identification of Tim4 as a phosphatidylserine receptor. Nature. 2007;450(7168):435-439.

56. Holland WL, et al. Receptor-mediated activation of ceramidase activity initiates the pleiotropic actions of adiponectin. Nat Med. 2011;17(1):55-63.

57. Holland WL, et al. Inducible overexpression of adiponectin receptors highlight the roles of adiponectin-induced ceramidase signaling in lipid and glucose homeostasis. Mol Metab. 2017;6(3):267-275.

58. Vasiliauskaité-Brooks I, et al. Structural insights into adiponectin receptors suggest ceramidase activity. Nature. 2017;544(7648):120-123.

59. Robbins PD, Morelli AE. Regulation of immune responses by extracellular vesicles. Nat Rev Immunol. 2014;14(3):195-208.

60. Lai RC, et al. Exosome secreted by MSC reduces myocardial ischemia/reperfusion injury. Stem Cell Res. 2010;4(3):214-222.

61. Lee C, et al. Exosomes mediate the cytoprotective action of mesenchymal stromal cells on hypoxia-induced pulmonary hypertension. Circulation. 2012;126(22):2601-2611.

62. van Balkom BW, et al. Endothelial cells require miR-214 to secrete exosomes that suppress senescence and induce angiogenesis in human and mouse endothelial cells. Blood. 2013;121(19):3997-4006.

63. Maeda N, et al. Diet-induced insulin resistance in mice lacking adiponectin/ACRP30. Nat Med. 2002;8(7):731-737.

64. Hebbard LW, Garlatti M, Young LJ, Cardiff RD, Oshima RG, Ranscht B. T-cadherin supports angiogenesis and adiponectin association with the vasculature in a mouse mammary tumor model. Cancer Res. 2008;68(5):1407-1416.

65. Mu J, Brozinick JT, Valladares O, Bucan M, Birnbaum MJ. A role for AMP-activated protein kinase in contraction- and hypoxia-regulated glucose transport in skeletal muscle. Mol Cell. 2001;7(5):1085-1094.

66. Théry C, Amigorena S, Raposo G, Clayton A. Isolation and characterization of exosomes from cell culture supernatants and biological fluids. Curr Protoc Cell Biol. 2006; Chapter 3:Unit 3.22

67. Kalra H, et al. Comparative proteomics evaluation of plasma exosome isolation techniques and assessment of the stability of exosomes in normal human blood plasma. Proteomics. 2013;13(22):3354-3364

68. Koyama Y, Nishida T, Tohyama M. Establishment of an optimised protocol for a Golgi-electron microscopy method based on a Golgi-Cox staining procedure with a commercial kit. J Neurosci Methods. 2013;218(1):103-109.

69. Lässer C, Eldh M, Lötvall J. Isolation and characterization of RNA-containing exosomes. J Vis Exp. 2012 ;(59):e3037.

70. Perry DK, Bielawska A, Hannun YA. Quantitative determination of ceramide using diglyceride kinase. Meth Enzymol. 2000;312:22-31.

71. Tani M, Okino N, Mitsutake S, Ito M. Specific and sensitive assay for alkaline and neutral ceramidases involving C12-NBDceramide. J Biochem. 1999;125(4):746-749.

72. Nikolova-Karakashian M, Merrill AH. Ceramidases. Meth Enzymol. 2000;311:194-201. 